\title{
The zebra finch auditory cortex reconstructs occluded syllables in conspecific song
}

Authors: Margot C Bjoring ${ }^{1}$ and C Daniel Meliza ${ }^{1,2, *}$

Affiliations: ${ }^{1}$ Department of Psychology, ${ }^{2}$ Neuroscience Graduate Program, University of Virginia, Charlottesville VA 22904, USA

* Corresponding author. Email: cdm8j@virginia.edu

Sensory input provides incomplete and often misleading information about the physical 1 world. To compensate, the brain uses internal models to predict what the inputs should be 2 from context, experience, and innate biases (Liberman and Mattingly, 1985; Komatsu, 2006; 3 Gilbert and Sigman, 2007; Heald and Nusbaum, 2014). For example, when speech is inter- 4 rupted by noise, humans perceive the missing sounds behind the noise (Miller and Licklider, 5 1950; Warren, 1970; Samuel, 1996), a perceptual illusion known as phonemic (or auditory) 6 restoration. The neural mechanisms allowing the auditory system to generate predictions that 7 override ascending sensory information remain poorly understood. Here, we show that the 8 zebra finch (Taeniopygia guttata) exhibits auditory restoration of conspecific song both in a 9 behavioral task and in neural recordings from the equivalent of auditory cortex. Decoding 10 the responses of a population of single units to occluded songs reveals the spectrotemporal 11 structure of the missing syllables. Surprisingly, restoration occurs under anesthesia and for 12 songs that the bird has not heard. These results show that an internal model of the general 13 structure of conspecific vocalizations can bias sensory processing without attention.

Auditory restoration is one of a number of ways the brain extracts signals of interest from complex 15 auditory scenes (Bregman, 1999). When phonemes are deleted from a speech stream and replaced 16 with noise, listeners hear the missing phonemes behind the occluding noise (Miller and Licklider, 17 1950; Warren, 1970). The restored phonemes are appropriate to their phonological and lexical 18 context (Warren and Sherman, 1974; Samuel, 1996), and restoration is stronger for words in the 19 
listener's native language compared to foreign or nonsense words (Samuel, 1996; Ishida and 20 Arai, 2016). These observations are consistent with cognitive models of speech perception in ${ }_{21}$ which top-down predictions based on context and experience actively influence processing in 22 primary auditory areas (Heald and Nusbaum, 2014; Leonard et al., 2016). Alternatively, recurrent ${ }_{23}$ connectivity within auditory areas may be such that local neural circuit dynamics are sufficient 24 to fill in missing information. Indeed, many illusions, including the visual analog of auditory 25 restoration, can occur without attention (Komatsu, 2006), so it is possible that expectations about 26 the acoustic structure of speech are implemented preattentively, within the auditory system.

Neural recordings have the potential to elucidate where and how auditory restoration occurs. In 28 humans presented with occluded speech, the auditory cortex exhibits illusory responses: patterns ${ }^{29}$ of activity that closely resemble those evoked by the phoneme the subject reports hearing (Leonard 30 et al., 2016). These recordings also show activity in higher-order language areas that precedes the 31 missing syllable and predicts perception, suggestive of top-down influences but not excluding local 32 computations. Nonhuman animals also experience a form of auditory restoration (Braaten and 33 Leary, 1999; Miller et al., 2001; Petkov et al., 2003; Seeba and Klump, 2009), but neural responses 34 have only been tested with simple tones (Sugita, 1997; Petkov et al., 2007), which lack the complex 35 acoustic structure of speech and may involve different mechanisms. We therefore turned to 36 the zebra finch, a social songbird with an acoustically rich song used for communication in 37 complex, noisy social environments (Singh and Theunissen, 2003; Elie and Theunissen, 2016). 38 After establishing that zebra finches experience illusory continuity using a behavioral task closely 39 modeled on human psychophysical studies (Samuel, 1996), we recorded neural responses to 40 occluded stimuli from the avian homolog of auditory cortex. Recordings were under anesthesia, 41 thereby silencing top-down processes requiring attention. We employed a linear decoding model 42 to test whether these neurons respond to the illusory percept and to determine where and when 43 the illusion emerges within the auditory processing hierarchy. 


\section{Zebra finches experience illusory continuity of occluded songs}

We tested the perception of auditory restoration in zebra finches using an oddball-detection paradigm (Fig. 1a). Birds were trained to detect a brief discontinuity in a sequence of otherwise identical conspecific song motifs. To create these discontinuities, we elided the sound in a 100ms critical interval, replacing it with white noise (Replaced condition). The other motifs in the sequence were continuous versions of the same motif with white noise added on top of the critical interval (Added condition). The amplitude of the white noise was varied so that the signal-to-noise ratio (SNR) ranged between 20 and $-15 \mathrm{~dB}$. The motifs were recorded from males in our colony, and the experimental birds were socialized with four of those males prior to training (Fig. 1b) so that half of the stimuli were familiar to them and half were unfamiliar.

At high SNR, trained birds $(n=5)$ were proficient at detecting the discontinuity, despite the difficulty of learning the task (7 of 12 birds were excluded for failing to reach baseline performance criteria). At $20 \mathrm{~dB}$ SNR, the mean hit rate was $0.50 \pm 0.07$ with a false alarm rate of $0.06 \pm 0.03$ (Fig. 1c). As the noise intensity within the critical interval increased, detecting the gap became more challenging, and hit rate decreased. The slope of the drop-off in hit rate was similar across all subjects, but there were individual differences in baseline performance and perceptual thresholds. The false alarm rate stayed constant across noise levels and varied little among subjects. It was also well below 0.2 , the prior probability that a given motif would contain a gap, indicating a strong bias against responding unless the bird was confident it could hear the discontinuity.

A hallmark of perceptual illusions is that performance in behavioral tasks drops below chance. This is because subjects are not merely guessing, but are fooled into perceiving something that 65 is not actually there (Samuel, 1996; Petkov et al., 2003). We therefore expected birds to make 66 systematic errors at noise levels that induce restoration. To test this prediction, we fit the data 67 with a generalized linear mixed-effects model (GLMM; see Materials and Methods) that included $\quad 68$ effects for familiarity, SNR, and the position of the oddball within the sequence. Although we were ${ }_{69}$ primarily interested in the effects of SNR and familiarity, there was a strong, nonlinear interaction 70 with position (Supplementary Fig. 2). Animals performed considerably better on the task when the 71 Replaced motif came last in the sequence, suggesting the influence of perceptual anchoring (Braida 72 
bioRxiv preprint doi: https://doi.org/10.1101/2021.07.19.452925; this version posted July 19, 2021. The copyright holder for this preprint (which was not certified by peer review) is the author/funder, who has granted bioRxiv a license to display the preprint in perpetuity. It is made available under aCC-BY-NC-ND 4.0 International license.

Bjoring and Meliza - Neural reconstruction of occluded song (preprint) p. 4
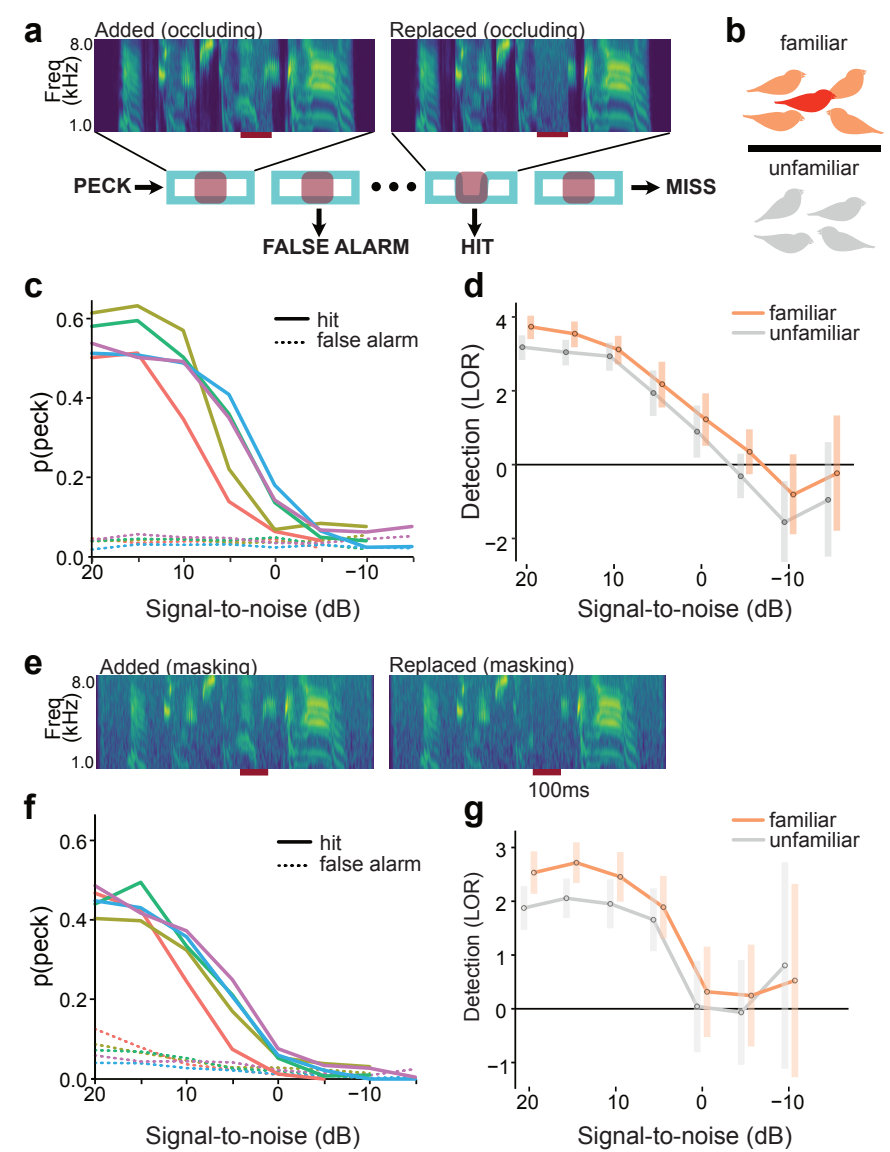

Fig. 1. Illusory perception of continuity during oddball detection task. (a) Example stimuli and trial structure for the oddball detection task with occluding noise. Red lines under the spectrograms indicate critical intervals. The position of the Replaced motif within the sequence was varied randomly. (b) Prior to the behavioral task, the subject (red) was familiarized with four of the males whose songs were used in the task (orange). The other four motifs were from birds with whom the subject had never been housed (gray). Familiarity was counterbalanced across subjects. (c) Average hit and false alarm rates for each of the subjects, with color indicating subject identity. (d) Estimates of average performance (log odds ratio, LOR) as a function of noise intensity and stimulus familiarity, with 0 corresponding to equal odds of pecking to Replaced and Added. Error bars show 90\% confidence intervals, and estimates are only for the first interval in which the oddball could appear (see Supplementary Fig. 2 for all intervals). Confidence intervals become wider with increasing noise due to fewer trials at higher noise levels. Ignoring familiarity, performance is significantly greater than chance with $\mathrm{SNR} \geq 0 \mathrm{~dB}(p<0.003)$ and worse than chance at $-10 \mathrm{~dB}$ SNR (LOR $=-1.19, z=-2.20, p=0.028,90 \% \mathrm{CI}=\{-2.24,-0.13\}$ ). Comparing familiar (orange) to unfamiliar motifs (gray), performance is better for familiar stimuli across all noise levels $\left(F_{1, \infty}=21.9, p<0.001\right)$, including specifically at $-10 \mathrm{~dB}(\mathrm{LOR}=0.74, z=2.49, p=0.013$, $90 \% \mathrm{CI}=\{0.25,1.23\}$ ). (e) Examples of masked stimuli. Noise was added to the entire motif instead of just the critical interval to eliminate the restoration illusion. (f) Hit and false alarm rates for masked stimuli. Hit rates were lower than for the occluded stimuli even at the highest signal-to-noise ratio (solid lines). False-alarm rates decreased as SNR decreased, indicating a different response strategy than for the auditory restoration task (dotted lines). (g) Estimates of average performance for masked stimuli. Task performance remains at or above chance for all SNR values for both familiar and unfamiliar stimuli. Data for $-15 \mathrm{~dB}$ SNR are omitted because the model could not otherwise converge, given the near-zero hit and false-alarm rates for that noise level. 
et al., 1998; Zokoll et al., 2007) or the use of alternative, guessing-based strategies (for example, 73 if no gap is heard in the first four motifs, then the conditional probability of it being in the last 74 motif is much higher than the marginal probability). Thus, the results presented here are taken 75 from the estimates of performance for the first interval when the gap could occur. This is the 76 most difficult condition, because the bird has the least information and will be least affected by 77 perceptual anchoring.

The effect estimates from the model provided strong evidence that zebra finches are susceptible 79 to the auditory restoration illusion (Fig. 1d). As expected, performance decreased as the noise 80 intensity increased, dropping significantly below chance to an estimated LOR of -1.19 at $-10 \mathrm{~dB} \quad 81$ SNR. On the response scale, this means the average bird was over 3 times less likely to peck to the $\quad 82$ Replaced stimulus than would be expected from the baseline false-alarm rate.

As a control, the auditory restoration test trials were interleaved with trials in which the noise 84 in the Added and Replaced conditions extended the entire length of the motif (Masked condition; 85 Fig. 1e). In human and other animal studies, this kind of masking does not induce an illusory 86 percept (Petkov et al., 2003), so performance is expected to drop to chance but not below. Overall, 87 zebra finches performed worse on Masked trials, and there was a tendency for the false alarm 88 rate to decrease at the loudest noise levels, perhaps because the noise itself was aversive (subjects 89 essentially refused to respond at all at the highest noise level). Importantly however, performance 90 on the masked task remained at or above chance (Fig. 1e,f).

In human studies, familiarity with the stimuli tends to strengthen the effect of phonemic restoration. $\quad 92$ Surprisingly, although we saw an effect of familiarity on performance, it was of the opposite sign 93 to what we expected. The birds had a harder time detecting gaps in unfamiliar stimuli across the 94 range of noise intensities, and performance dropped off more quickly as SNR decreased (Fig. 1d). 95 At $-10 \mathrm{~dB}$ SNR, although there was still a clear trend for performance to be worse than chance, the 96 estimated LOR for familiar stimuli was not significantly less than zero (LOR $=-0.81, z=-1.47, \quad 97$ $p=0.14)$.

Overall, we found that by manipulating zebra finch song following the principles of phonemic $\quad 99$ restoration, we could detect behavioral evidence of an illusory percept of continuity. The perfor- 100 
mance of zebra finches trained to detect discontinuities in conspecific song dropped significantly 101 below chance as the intensity of the occluding noise increased, while their performance on control 102 stimuli designed not to induce an illusory percept dropped to chance but not below.

\section{Neural responses to the illusory percept}

The behavioral results imply that at some level of the zebra finch's auditory system, neurons 105 are responding to an illusion rather than to the stimulus that was physically present. To test 106 this hypothesis, we made extracellular recordings of 407 single units ( $n=14$ birds) across the ${ }_{107}$ avian auditory cortex, including the caudal mesopallium (CM, $n=56$ units), field L subunits L1 108 ( $n=25$ units), L2a ( $n=33$ units), and L3 ( $n=59$ units), and the caudomedial nidopallium (NCM, 109 $n=90$ units) (Fig. 2a). We made these recordings under anesthesia to further test whether illusory ${ }_{110}$ responses require top-down processing.

Zebra finches were presented with the illusion-inducing Replaced stimulus (RS) as in the behavioral 112 experiment and with three control variants: the Continuous unmodified stimulus (CS); a Discontin- 113 uous stimulus (DS) where the note in the critical interval was elided but not replaced by any noise; 114 and a Noise-only stimulus (NS) (Fig. 2b). Note that the CS and DS stimuli are similar to Added and 115 Replaced, respectively, with noise at the lowest level.

We observed a wide variety of response patterns to these stimuli. Many individual neurons were ${ }_{117}$ highly sensitive to elision of the critical interval (DS) but responded to the RS stimulus as if it were ${ }_{118}$ continuous (Supplementary Fig. 5a,b). Across the population, each unit's average firing rate during ${ }_{119}$ the critical interval of the RS stimuli was nearly identical to its response in the corresponding 120 interval of the CS stimuli, such that almost $90 \%$ of the variance in RS response rates could be ${ }_{121}$ predicted by the responses to CS stimuli. In contrast, the firing rates elicited by RS stimuli were 122 significantly less like the responses to DS and NS (Supplementary Fig. 5c,d).

To test whether these similarities were indicative of an illusory response (i.e., a pattern of activity 124 similar to the response evoked by the missing syllable), we used a linear decoder to predict ${ }_{125}$ what stimulus was being presented from the responses to the RS motifs. The parameters of the 126 decoder were fit using ridge regression on data from the three control variants (CS, DS, and NS) ${ }_{12}$ 
bioRxiv preprint doi: https://doi.org/10.1101/2021.07.19.452925; this version posted July 19, 2021. The copyright holder for this preprint (which was not certified by peer review) is the author/funder, who has granted bioRxiv a license to display the preprint in perpetuity. It is made available under aCC-BY-NC-ND 4.0 International license.

Bjoring and Meliza - Neural reconstruction of occluded song (preprint) p. 7

a

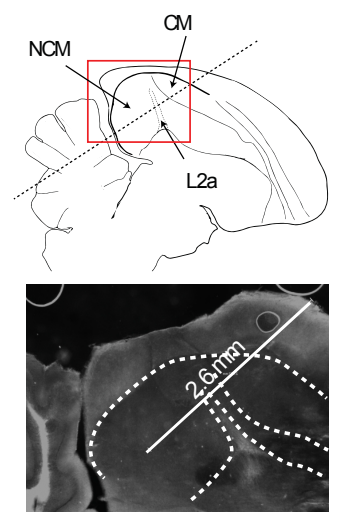

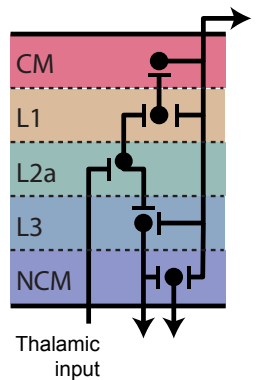

b

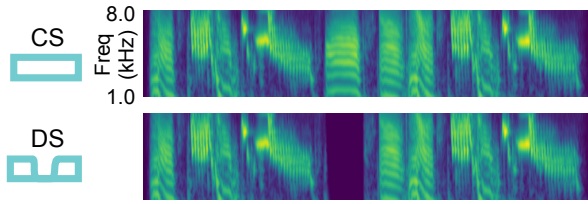

NS

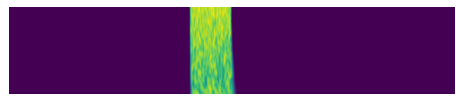

RS

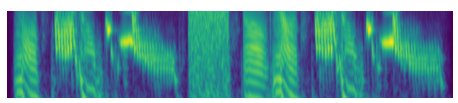

$\mathrm{C}+\mathrm{N}$

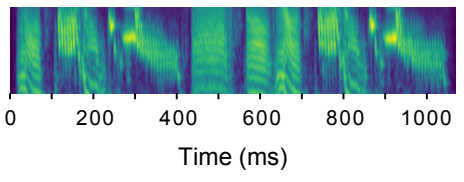

e
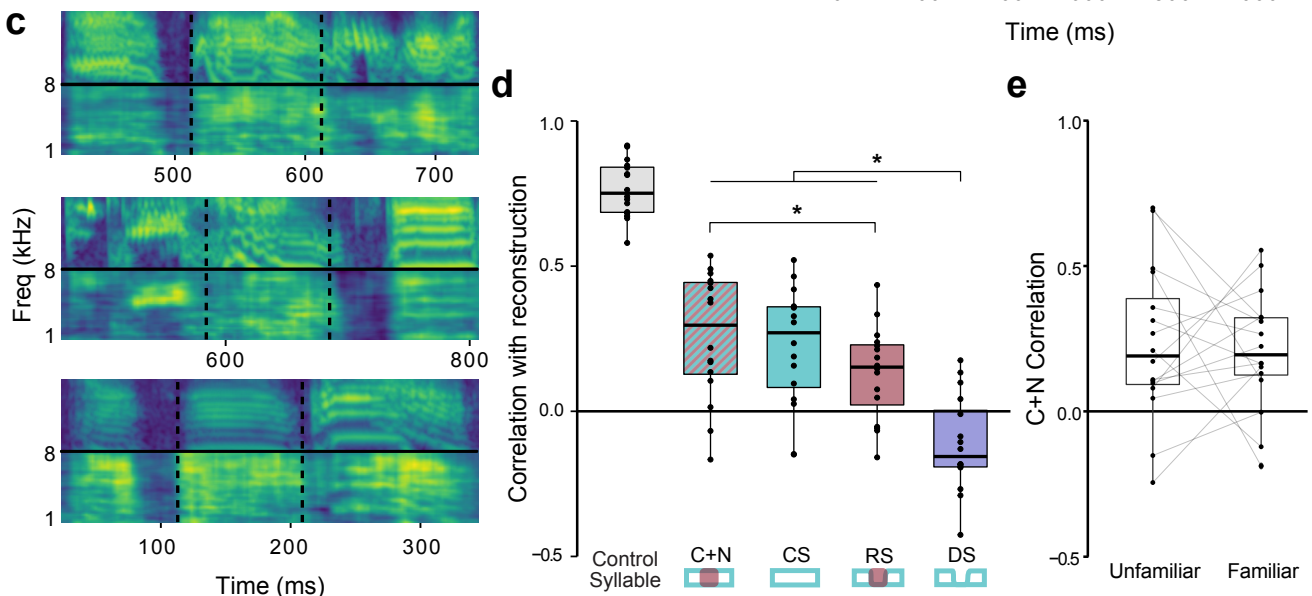

Fig. 2. Neural responses to occluded stimuli. (a) Location and microcircuitry of recording site. Counterclockwise from top: saggital diagram of zebra finch brain, with dashed line showing approximate orientation of the recording probe (adapted from Chen and Meliza, 2018); micrograph of auditory cortex showing DiI tracks; diagram of the subdivisions of the avian auditory cortex and their interconnections (adapted from Calabrese and Woolley, 2015). (b) Spectrograms of the four variants of an example motif used in extracellular recordings and a fifth variant used in analyses only $(\mathrm{C}+\mathrm{N})$. CS: continuous, unmanipulated motif; DS: discontinuous, with critical syllable deleted; NS: noise, a burst of white noise with the same duration as the critical syllable; RS: replaced, consisting of NS added to DS; $\mathrm{C}+\mathrm{N}$ : an approximation of the expected illusory percept combining the CS syllable and the NS noise in the critical interval. (c) Reconstruction of three exemplar motifs from responses to RS stimuli. In each panel, the top row is a spectrogram of the CS stimuli surrounding the critical interval (dotted vertical lines), and the bottom row is the reconstruction. Times are relative to the start of the motif. (d) Similarity (correlation coefficient) of the reconstructed stimuli to the presented stimuli (CS, DS, and RS) and the expected illusory percept $(\mathrm{C}+\mathrm{N})$. The control syllable was outside the critical interval and was the same for CS, DS, and RS. The other groups show similarity for the critical interval in each of the four variants. Individual points and lines correspond to motifs. Boxplots show medians (horizontal line), interquartile ranges (boxes), and 10-90 percentile ranges (whiskers). Reconstructions were more similar to $\mathrm{C}+\mathrm{N}$ than to RS (Kenward-Roger: $t_{60}=2.24, p=0.03$ ). The difference in similarity to CS and RS was not significant $\left(t_{60}=1.59, p=0.12\right)$. Similarity to DS was lower than to $C+N, C S$, and $\operatorname{RS}(p<0.0001)$. (e) Data were split by stimulus familiarity and the decoder was fit separately to each subset. Individual points show correlation coefficient between reconstructions and the $\mathrm{C}+\mathrm{N}$ variant (as in $\mathbf{b}$ ), with lines connecting each stimulus from the subset where it was unfamiliar to the subset where it was familiar. Across all stimuli, there was no significant effect of familiarity on reconstruction quality (paired t-test: $\left.t_{28}=0.29, p=0.77\right)$. 
and then used to reconstruct the expected stimulus from the RS responses. The decoder yielded 128 reconstructions that appeared to be a superposition of the missing syllable and the occluding noise, 129 as if both the physical stimulus and the illusion were simultaneously present. In some cases the 130 reconstruction looked remarkably similar to the CS motif (Fig. 2c, top panels), and in other cases 131 the noise appeared to dominate (Fig. 2c, bottom panel). However, even the noisy reconstructions 132 had spectrotemporal structure that bore some resemblance to the missing syllable.

To quantify similarity, we calculated the correlation coefficient between the reconstructed spec- 134 trogram and the spectrograms of the corresponding CS, DS, and RS variants (Fig. 2d). We also 135 compared the reconstructions to a synthetic variant $(\mathrm{C}+\mathrm{N})$ comprising a 1:1 mixture of the missing $\quad{ }_{136}$ syllable and the occluding noise (Fig. 2b, bottom). The baseline performance of the decoder was ${ }_{137}$ excellent despite its simplicity: for an unmanipulated syllable outside the critical interval, the 138 correlation between the prediction and the actual stimulus was high $(r=0.85 \pm 0.05)$. Within 139 the critical interval, the reconstructions were more similar to the $\mathrm{C}+\mathrm{N}$ spectrograms than to the 140 RS spectrograms, indicating that the population activity contains information about the acoustic 141 structure of the occluded syllable, even though it was not physically present in the stimulus. The ${ }_{142}$ reconstructions were also more similar to the $\mathrm{C}+\mathrm{N}, \mathrm{CS}$, and RS spectrograms than to DS, showing ${ }_{143}$ strong evidence against discontinuity.

As in the behavioral experiments, familiarity was counterbalanced such that half of the birds ${ }_{145}$ we recorded from were familiar with a different half of the motifs (Fig. 1b). We tested whether ${ }_{146}$ reconstruction was affected by familiarity by fitting the decoder separately to neurons from each 147 of the two groups of subjects. Thus, one model was "familiar" with half of the motifs while the 148 other was familiar with the other half. For each motif, we then compared the reconstructions 149 from the familiar and the unfamiliar model to $\mathrm{C}+\mathrm{N}$. The average difference was not statistically 150 distinguishable from zero (Fig. 2d). Thus, the avian auditory cortex is apparently able to reconstruct 151 the acoustic structure of occluded syllables in songs that a bird has never heard. 

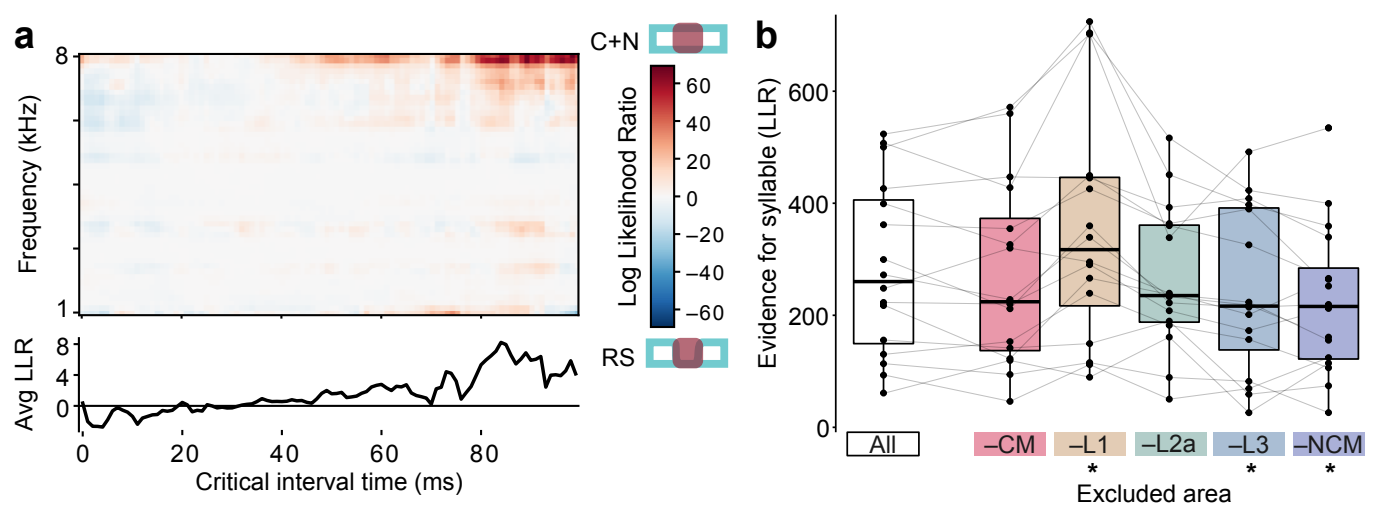

Fig. 3. Evidence for the missing syllable increases with time and is distributed throughout the cortical hierarchy. (a) Log of the posterior likelihood ratio for $\mathrm{C}+\mathrm{N}$ over RS during the critical intervals as a function of frequency and time, averaged across all stimuli. Red indicates that given the response, the stimulus was more likely to be $\mathrm{C}+\mathrm{N}$ than $\mathrm{RS}$, and blue indicates $\mathrm{RS}$ was more likely than $\mathrm{C}+\mathrm{N}$. Bottom trace shows the average across all frequencies. (b) Change in the log likelihood ratio of $\mathrm{C}+\mathrm{N}$ vs RS after fitting decoding models to data with all the units from one area left out compared to the evidence for $\mathrm{C}+\mathrm{N}$ against the full model (see Materials and Methods). Note the sign reversal: a decrease in LLR indicates that the area was contributing evidence toward C+N. L1 units reduced the evidence for $\mathrm{C}+\mathrm{N}$ relative to the full model $\left(p<0.001, t_{60}=-6.55\right)$, and $\mathrm{L} 3$ and NCM units increased the evidence for $\mathrm{C}+\mathrm{N}\left(p=0.034, t_{60}=2.38\right.$ and $p=0.002, t_{60}=3.59$, respectively).

\section{Responses become more illusory over the occluded interval}

We hypothesize that the illusory percept of the missing syllable is generated by a predictive internal $\quad 154$ model and that the neural activity associated with this internal model is in conflict with ascending 155 sensory input. The strength of the illusion could therefore depend on how effectively the response 156 to noise is suppressed, and suppressing this activity could take time. To test this idea, we used ${ }_{157}$ the decoding model to calculate the amount of evidence in the neural response for the missing syllable. We quantified this as the log ratio of the (posterior predictive) likelihood that the presented 158 stimulus was $\mathrm{C}+\mathrm{N}$ over the likelihood that it was $\mathrm{RS}$, for each time point and spectral channel during the critical interval (Fig. 3a).

Consistent with our hypothesis, we saw a strong change in the evidence for the missing syllable over 162 the course of the critical interval. Initial responses were biased towards noise, perhaps reflecting the 163 appearance of a new and potentially salient signal. By $20 \mathrm{~ms}$, however, the evidence shifted toward 164 the missing syllable and continued to strengthen over the course of the critical interval, supporting 165 the view of a predictive internal model driving the neural response after the initial onset of noise. ${ }^{166}$ 
evidence for the missing syllable in the highest frequencies near the limit of the zebra finch's 168 hearing range (Fig. 3a). This result likely reflects the fact that the biggest differences in the power 169 spectra of zebra finch song and white noise are in the higher frequencies (compare NS to CS in 170 Fig. 2b). There were also distinct bands of evidence at lower frequencies, which could correspond 171 to species-typical spectral structure.

\section{Illusory responses are distributed throughout the auditory pallium}

Where is the illusion of the missing syllable created? The avian auditory cortex comprises several 174 laminar subdivisions organized in a hierarchy of increasingly complex functional properties (Sen 175 et al., 2001; Meliza and Margoliash, 2012; Calabrese and Woolley, 2015). The illusion could be 176 generated in one of these areas and then propagate to downstream areas. Alternatively, it could 177 emerge in a more distributed manner or propagate from higher-order areas through feedback. To 178 help distinguish between these possibilities, we tested how removing each of the five areas from 179 the decoder impacted the evidence for the missing syllable. The advantage of this subtractive 180 approach is two-fold: first, it allowed for similar numbers of units in each decoder, producing ${ }_{181}$ reconstructions comparable in quality to the full model; and second, it allowed us to identify the ${ }_{182}$ unique contributions of each auditory area. Because the decoding model accounts for correlated 183 activity between neurons, removing redundant units will result in a shift of the linear weights to ${ }_{184}$ other cells, with little change to the model estimates. Therefore, if the posterior predictive likelihood 185 of $\mathrm{C}+\mathrm{N}$ decreases relative to $\mathrm{RS}$ after removing data from one area, we can conclude that area is 186 making a non-redundant contribution to the illusory response.

With the full model, the evidence from the neural response was strongly in favor of the presence of ${ }_{188}$ the missing syllable for every motif tested (Fig. 3b). This result is consistent with the spectrogram 189 correlations (Fig. 2B), but quantitatively different because the likelihood ratio accounts for posterior 190 uncertainty. Removing specific areas from the model did not change the overall picture: evidence 191 was still overwhelmingly for the missing syllable. However, some areas made significant, non- 192 redundant contributions to the evidence for (or against) the missing syllable. L3 and NCM both 193 increased the likelihood of $\mathrm{C}+\mathrm{N}$ relative to RS, whereas L1 biased the reconstruction more toward 194 the noise (Fig. 3b). CM and L2a did not make a statistically significant contribution in either 195 
direction. Thus, although the illusory response appeared to be distributed throughout the auditory 196 cortex, the deeper subdivisions (Fig. 2a) were the most responsive to the illusion compared to the 197 physical stimulus.

\section{Discussion}

Perceptual illusions present a unique opportunity to expand our knowledge of how the brain 200 uses internal models in sensory processing. Normally, internal models are aligned with incoming 201 sensory input, but in an illusion, perception diverges from reality, and the neural activity produced 202 by a model can be dissociated from the activity produced by the physical stimulus. In this study, 203 we saw both behavioral and direct neural evidence that the zebra finch auditory cortex has an 204 internal model of how conspecific songs should sound. This model biases auditory responses even 205 under anesthesia and appears to be based on the general acoustic structure of song rather than 206 specific memories.

The perceptual illusion we observed is analogous to phonemic restoration in humans (Warren, 1970; 208 Warren and Sherman, 1974; Samuel, 1996; Bregman, 1999; Ishida and Arai, 2016). As in humans, it 209 occurs when the occluding noise is brief and about the same intensity as the vocalization. Like all 210 illusions, it is characterized by performance that becomes worse than chance, a sign that the subject 211 is not simply guessing in the absence of evidence but convinced to some degree that the illusory 212 percept is real (Petkov et al., 2003). By comparison, we found that when noise encompasses the ${ }_{213}$ entire stimulus, obscuring not only the gap but the all the acoustic features of the motif, performance 214 remains at or above chance. These results are similar to those obtained in macaques using pure 215 tones (Petkov et al., 2003) and consistent with observations in European starlings using a simpler ${ }_{216}$ and less stringent test (Seeba and Klump, 2009), but not with behavior in frogs (Seeba et al., 2010). 217

The strongest evidence for auditory restoration in zebra finches is that the brain responds as if ${ }_{218}$ the missing syllable were present (Bregman, 1999). We were able to detect this using a simple ${ }_{219}$ decoding model consisting of the best linear projection from the pattern of neural activity in the 220 population to the power spectrum of the stimulus at a given instant in time (Mesgarani et al., 221 2009; Crosse et al., 2016). Note that the decoder has no information about the structure of the ${ }^{222}$ 
stimulus except via the neural response. Moreover, it is simply a tool to analyze the patterns of ${ }^{223}$ activity in the population, not a hypothesis about the computation that allows the brain to infer ${ }^{224}$ or predict what incoming sensory inputs should look like. More complex, nonlinear decoders 225 will surely do a better job of prediction, but at the cost of introducing more untested assumptions, 226 and with a greater risk of overfitting (Glaser et al., 2020). We minimized overfitting here by using 227 regularization and by training the model on responses to a large corpus of birdsong and noise ${ }_{228}$ bursts while holding out the trials with occluded stimuli for testing. When we used the model to 229 infer what stimulus is being presented during the occluded portion of a song, the distribution of 230 likely stimuli is overwhelmingly biased toward a mixture of the noise that was physically present ${ }^{231}$ and the syllable that should have been there. Human subjects report hearing a similar mixture of 232 illusory and physical percepts in phonemic restoration.

In humans, the sensitivity of phonemic restoration to lexical and phonological context (Warren 234 and Sherman, 1974; Samuel, 1996) is suggestive of top-down modulation actively shaping how 235 lower-level auditory areas process ambiguous inputs. Such processes are generally assumed to 236 be active, requiring attention and other cognitive resources (Heald and Nusbaum, 2014), but our 237 results show this is not necessarily the case. Our observation of illusory responses in anesthetized ${ }_{238}$ animals suggests that local circuit dynamics can fill in missing information. These dynamics could 239 be entrained by a lifetime of experience with zebra finch song through Hebbian plasticity of the 240 dense network of recurrent connections within the auditory pallium (Wang et al., 2010; Calabrese ${ }^{241}$ and Woolley, 2015) and could bias the response of the network towards familiar or species-typical 242 patterns. We observed this bias emerge quickly but not instantaneously: the response to the 243 ambiguous stimulus segment is initially more consistent with noise but shifts to the missing 244 syllable after about $20 \mathrm{~ms}$, increasing throughout the critical interval (Fig. 3a). We hypothesize that 245 peripheral adaptation to broadband noise may allow the cortical network dynamics to gradually 246 dominate. These dynamics may be able to persist on their own for some time: in humans, the ${ }_{247}$ limit of illusory continuity in speech is approximately $300 \mathrm{~ms}$ (Bashford and Warren, 1987), and 248 further work could characterize the full time-course of the neural illusion by recording responses 249 to increasingly longer occlusions. 
familiarity with specific songs for auditory restoration to occur. European starlings show evidence 252 of illusory continuity only for familiar stimuli (Braaten and Leary, 1999; Seeba and Klump, 2009), 253 and in humans, phonemic restoration is stronger for words in the listener's native language 254 compared to foreign or nonsense words (Samuel, 1996). These findings indicate that experience 255 shapes the brain's internal models of what is being occluded by the noise. However, listeners do 256 restore phonemes in non-native speech, just to a lesser degree (Ishida and Arai, 2016), suggesting 257 that the internal models are not simply memorized images of specific phonemes or syllables. 258 Instead, the auditory cortex may have a more general model of conspecific syllable and spectral ${ }^{259}$ patterns, and it may even be filling in a superposition of multiple candidates, as has been proposed 260 for human speech recognition in the Trace model and its successors (McClelland and Elman, 1986; 261 Heald and Nusbaum, 2014). Compared to starlings, zebra finches have a fairly limited set of vocal 262 gestures, so it may be easier for them to learn a more general model of conspecific song. This effect ${ }_{263}$ may have been magnified in these experiments because all the songs came from the same colony. $\quad 264$

We found evidence for the missing syllable distributed over all the areas we recorded, though the 265 deeper auditory areas L3 and NCM show a somewhat greater bias towards the missing syllable 266 (Fig. 3b). NCM is a secondary auditory area with high selectivity for conspecific song (Meliza and ${ }^{267}$ Margoliash, 2012), and it contains a subset of noise-invariant neurons (Schneider and Woolley, 268 2013) that could play a prominent role in sustaining the response to song through the occluding 269 noise. Nevertheless, the entire auditory pallium appears to respond to the illusory syllable, even 270 the thalamorecipient area L2a, which has highly linear response properties (Calabrese and Woolley, 271 2015). This implies that all these areas either participate in computing the illusory response or 272 respond to recurrent connections from the neurons that do. The present study aggregates data from 273 multiple subjects and averages across trials, obscuring individual- and trial-level dynamics that 274 may yield more insight into the origin of the illusory activity. Recordings from animals engaged in 275 the behavioral task could also reveal patterns of activity and specific areas that predict whether an 276 individual animal responds to the illusion (Leonard et al., 2016).

Although language is a uniquely human behavior, these results imply that other animals employing 278 complex vocalizations for communication have evolved similar mechanisms to compensate for 279 acoustic noise and interference. Indeed, the use of internal models that actively modulate neural 280 
activity at early stages of processing (Petkov and Sutter, 2011; Heald and Nusbaum, 2014) may be 281 a general feature of neural systems (Rao and Ballard, 1999). The same internal models may also 282 account for how populations of neurons throughout the avian auditory cortex are able to efficiently 283 filter out "cocktail-party" noise from conspecifics (Narayan et al., 2007; Schneider and Woolley, 284 2013). Given the link between poor speech perception in noise and a number of learning disabilities 285 (Bradlow et al., 2003), auditory restoration in zebra finches represents a powerful experimental 286 system to investigate how internal models for acoustic communication are formed and tuned by ${ }_{287}$ experience.

\section{Materials and methods}

\section{Animals}

All animal use was performed in accordance with the Institutional Animal Care and Use Committee 291 of the University of Virginia. Adult zebra finches were obtained from the University of Virginia 292 breeding colony. Eight zebra finches (all male) were used for song familiarization, 12 (5 female) ${ }_{293}$ were trained on the behavioral experiment, and 14 (7 female) were used for extracellular recording. 294

\section{Song recording and social familiarization}

Recordings were made of the songs of eight adult male zebra finches to use as stimuli in the 296 behavioral and electrophysiological experiments. Each singer was housed individually in a sound 297 isolation box (Eckel Industries, Cambridge, MA) with ad libitum food and water on a 16:8 h 298 light:dark schedule. A lavalier microphone (Audio-Technica Pro 70) was positioned in the box 299 near a mirror to stimulate singing. The microphone signal was amplified and digitized with 300 a Focusrite Scarlett $2 \mathrm{i} 2$ at $44.1 \mathrm{kHz}$, and recordings to disk were triggered every time the bird 301 vocalized using Jill (https://github.com/melizalab/jill; version 2.1.4), a custom C++ real-time 302 audio framework. A typical recording session lasted 1-3 days. From each bird's recorded corpus, a 303 single representative motif was selected and high-pass filtered using a 4th-order Butterworth filter 304 with a cutoff frequency of $500 \mathrm{~Hz}$. 
Subsequent to song recording, the eight males were randomly assigned to two groups of four and 306 housed in group cages in separate rooms in the breeding colony. Experimental birds were housed 307 in one of the group cages for at least one week to become familiar with the songs of the recorded 308 males (Fig. 1B). Experimental birds were assigned essentially at random, but with the constraint 309 that they had no prior social contact with the males in the group cage that they were not placed 310 in. Thus, familiarity was counterbalanced, with half of the motifs familiar to a different half of the 311 experimental subjects.

\section{Behavioral experiment}

Operant apparatus Behavioral experiments were run on a single-board computer (Beaglebone 314 Black) with a custom expansion board (https://meliza.org/starboard, revision A2A) that in- 315 terfaced with the operant manipulanda, cue lights, house lights, and feeder. The experiments 316 were implemented using an event-driven framework our lab has developed for controlling 317 behavioral experiments (https://github.com/melizalab/decide; version 3.2.1). Each subject 318 was housed individually in an acoustic isolation box (Eckel Industries) with its own appara- 319 tus and single-board computer, which sent the trial data it collected to a centralized database 320 (https:/ / github.com/melizalab/django-decide-host; version 0.3.0).

Acoustic stimuli were presented by the single-board computer through an Altec Lansing Orbit 322 iML227 USB speaker. The subject interacted with the apparatus by pecking an opening in a custom 323 printed circuit board fitted with infrared beam-break detectors and cue lights. Reinforcement was 324 standard finch seed, delivered through a custom 3D printed inlet housing a motorized screw shaft, 325 which was advanced by a stepper motor for 500 ms to deliver approximately 3 seeds (median; 326 range $0-13)$.

Shaping Following social exposure, behavioral subjects were moved to an acoustic isolation 328 box and allowed to acclimate for 1-3 days. Throughout the subsequent shaping, training, and 329 testing stages of the experiment, birds were maintained in the box on an semi-open economy. They 330 received seed from the feeder at 5-10 minute intervals throughout the day, but the feeder was shut ${ }_{331}$ off at least 30 minutes before beginning a training or testing session. During sessions, food was 332 
only available by completing trials. Behavior was monitored to ensure birds received adequate 333 food, feeding intervals were adjusted to ensure the birds maintained surplus seed, and sessions 334 were terminated if the bird went for more than 4 hours without eating.

Subjects were trained to peck the response panel using a standard autoshaping paradigm. First, a 336 cue light located near the opening was lit just before automatic food delivery. Once the bird started 337 pecking at the opening in anticipation of food, automatic food delivery stopped, and reinforcement 338 was only given after pecking. It was often helpful to suspend a small piece of string in the opening 339 during this initial shaping stage to encourage exploration. There were three blocks of 100 trials: in 340 the first block, the bird had to peck the lit opening once; in the second, it had to peck twice; and 341 in the third, the bird had to peck twice, but the cue light was eliminated. One of the 12 birds was 342 excluded during this stage because it failed to acquire the pecking behavior.

Stimuli For each of the eight motifs, eight variants were constructed using a $2 \times 2 \times 2$ design 344 (fig. 1). The first factor was the timing of the critical interval chosen for manipulation. Two non- 345 overlapping intervals were chosen for each motif, avoiding both the first and last notes of the motif. 346 The second factor was whether the motif was continuous or discontinuous. The discontinuous 347 variants were constructed by deleting the sound in the critical interval. The third factor was the 348 duration of white noise added to the motif. In the occluding case, the noise was only present during 349 the critical interval; in the masked case, the noise was present throughout the motif. Following 350 Petkov et al. (2003), edge artifacts were minimized by applying a $3 \mathrm{~ms}$ cosine ramp to the onsets 351 and offsets of the noise and the gaps in the occluding case. In the masked case, noise onset and 352 offset used a $25 \mathrm{~ms}$ cosine ramp.

The stimuli were amplified so that the unmodified motifs all had a RMS amplitude of $50 \pm 2 \mathrm{~dB} \quad 354$ SPL at the location where the bird interacted with the operant response panel, as measured by 355 an NTi Audio XL2 Sound Level Meter. For each variant, the amplitude of the white noise was 356 varied relative to the song in $5 \mathrm{~dB}$ increments between 20 and $-15 \mathrm{~dB}$ signal-to-noise ratio (SNR), 357 corresponding to SPLs between 30 and $65 \mathrm{~dB}$. Thus, there were a total of 512 different stimuli. For 358 an example of the full set of stimuli generated for one motif, see fig. 1. 
Behavioral task Zebra finches had to be trained in several stages to learn the experimental task. 360 In the first stage, the bird pecked to initiate a trial in which a single song motif was presented. A 361 second peck within $1 \mathrm{~s}$ of the end of the motif was rewarded. In the second stage, the bird listened 362 to two motifs and was rewarded for withholding a peck until the second motif was presented. 363

After shaping was completed, birds were trained on the main task, which was to detect a motif 364 with a gap in a sequence of otherwise identical motifs without gaps (Fig. 1A), presented with 365 $200 \mathrm{~ms}$ inter-stimulus intervals. The position of the discontinuous motif in the sequence was 366 random, but the first motif was always continuous. The finches had a short window from the 367 start of the gap in the discontinuous motif to peck for a correct response, which was rewarded 368 with seed. Pecking at any other time during the trial was a false alarm, and failure to peck when 369 there was a discontinuous motif was a miss. False alarms and misses were punished with a 2370 S "time-out" during which the house lights were extinguished and trials could not be initiated. 371 Stimulus playback ended immediately after a peck response, whether correct or incorrect. On 20\% 372 of the trials, all of the motifs were continuous, so the correct response was to withhold a peck, and 373 a reward was delivered at the end of the motif set. Supplementary Movie 1 is an annotated video 374 of a well-trained bird performing the task.

The initial training only used familiar motifs, and only the variants with occluding noise. To make 376 the starting difficulty as low as possible while still allowing the birds to learn the task, the SNR was 377 $20 \mathrm{~dB}$, the critical interval was $200 \mathrm{~ms}$, the sequence was three motifs long, and the finches had a $2 \mathrm{~s} 378$ window from the start of the gap in the discontinuous motif to peck for a correct response, which 379 was rewarded with food. The difficulty of the task was progressively increased by incrementally 380 shortening the response window from $2 \mathrm{~s}$ to $1 \mathrm{~s}$, then reducing the critical interval from $200 \mathrm{~ms}$ to 381 $150 \mathrm{~ms}$ to $100 \mathrm{~ms}$, then increasing the number of motifs from 3 to 4 to 5 . For the purpose of tracking 382 performance, the log-odds ratio (LOR) was calculated empirically from sliding blocks of 50 trials as 383

$$
L O R=\frac{1}{2} \log \left(\frac{H}{M} \times \frac{C R}{F A}\right)
$$

where $H$ is the proportion of trials with hits, $M$ is the proportion with misses, $C R$ is the proportion 384 with correct rejections, and FA is the proportion with false alarms. Note that this LOR is calculated 385 
on a per-trial basis and does not account for the fact that on many trials, birds have to correctly 386 reject multiple Added stimuli. To be included in the study, birds had to achieve and maintain 387 performance above an LOR of 1 . Birds that failed to show a systematic increase in performance 388 over a two week period were excluded. Five of the 12 birds were excluded during task-specific 389 shaping. The remaining six subjects learned the task within $15700 \pm 7800$ trials and achieved a final 390 LOR of $1.15 \pm 0.09$ (see table 1 for full breakdown of trials).

Five of the 12 birds had previously been trained on a similar oddball detection task where the 392 oddball was an entirely different motif. Of those birds, three successfully learned the new task and 393 were included in this study, and two were excluded during task-specific shaping.

\section{Testing auditory restoration}

Auditory restoration is characterized by an illusion of continuity when a gap in a stimulus is 396 occluded by noise of similar amplitude. Thus, as the noise amplitude increases, the odds of 397 responding during the correct interval is expected to decline while the false alarm rate remains 398 constant. We tested this prediction by systematically varying the amplitude of the occluding noise 399 while holding the motif amplitude constant, so that SNR ranged from 20 to $-15 \mathrm{~dB}$. The noise level 400 was the same for all motifs within a trial. Trials with louder noise were introduced in stepwise 401 blocks. That is, we started with all the trials at $20 \mathrm{~dB}$ SNR, then switched to a new block with $30 \% \quad 402$ trials at $15 \mathrm{~dB}$ SNR. In the next block, there were $22 \%$ trials at $15 \mathrm{~dB}, 22 \%$ trials at $10 \mathrm{~dB}$, and so forth. 403 We stopped on the block where the lowest SNR was $0 \mathrm{~dB}$ (50 dB SPL) or where the performance ${ }_{404}$ on the lowest-SNR stimuli dropped below chance, whichever came later. This procedure ensured 405 that a large proportion of the trials in each block were relatively easy for the bird, which helped to 406 maintain baseline performance and reduced the likelihood that birds would become frustrated and 407 switch to a guessing-based strategy.

During testing sessions, the unfamiliar motifs were included for the first time, and trials with 409 occluded variants were randomly interleaved with trials with masked variants. The noise intensity 410 distribution in the masked trials was matched to that of the occluded trials. Masking noise is an ${ }^{411}$ important control, because it also makes it more difficult for the birds to detect gaps in the motif, 412 but without inducing an illusion of continuity, so the decline in performance with noise amplitude ${ }_{413}$ 
is expected to be shallower. Moreover, without an illusory percept to fool the bird into thinking the ${ }_{414}$ stimulus is continuous, performance is expected to decline to chance but not below it.

Data analysis The data from the behavioral experiment comprised a total of 63,134 trials from 416 six birds. After an initial exploratory analysis, we excluded one bird because its false alarm rate on ${ }_{417}$ the catch trials (with no gap) was greater than its hit rate on the other trials. This left 50,809 trials ${ }_{418}$ (range 5085-13,611 per bird). Each trial was split into 1-5 intervals, one for each motif the bird ${ }_{419}$ heard (recall that trials terminated immediately after the bird responded). Each interval was coded ${ }_{420}$ with a single binary dependent variable, peck, which was 1 if the bird pecked during the interval ${ }_{421}$ and 0 otherwise, and with seven independent variables: $d B$, the amplitude of the noise (coded as a 422 factor with 8 levels); gap, the presence of a gap in the motif; position, the position of the interval ${ }_{423}$ in the sequence (coded as a factor); familiarity, whether the subject had been exposed to the motif ${ }_{424}$ in social housing; condition, whether the noise was occluding or masking; bird, the identity of the ${ }_{425}$ subject (coded as a factor); and song, the identity of the motif variant (two gap positions per motif, 426 coded as a factor).

A generalized linear mixed-effects model (GLMM) was used to infer the effects of noise intensity ${ }^{428}$ and familiarity on the subjects' ability to detect the motif with a gap in it. Peck was modeled as ${ }_{429}$ a binomial random variable with log odds that depended on a linear function of $d B$, gap, position, 430 familiarity, and their interactions. Two of the higher-order interactions (dB:position:familiarity and 431 dB:gap:position:familiarity) had to be removed for the parameter estimation to converge. Random ${ }^{432}$ effects were included to account for variations in psychophysical curves associated with subject ${ }_{433}$ and motif identity (fig. 3 and fig. 4), with motif identity nested in subjects. The parameters were ${ }^{434}$ estimated in R using lme4 (version 1.1-23). The model specification was as follows: 435

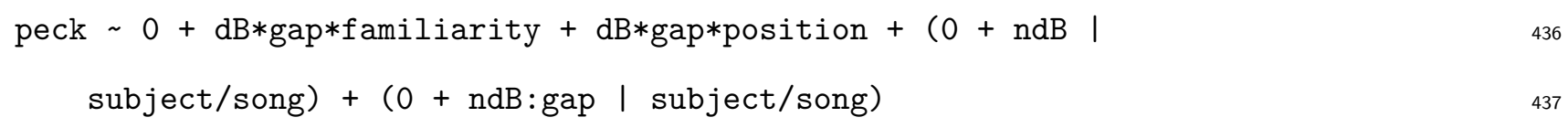

In this formulation, the parameters have the following interpretations: $d B$ gives the false alarm ${ }^{438}$ odds for unfamiliar stimuli at each of the eight noise levels; $d B$ :gap gives the log odds ratio of ${ }^{439}$ pecking when a gap is actually present, again for unfamiliar stimuli and at each of the noise levels; 440 $d B$ :familiarity gives the effect of familiarity on the log odds of false alarms; and dB:gap:familiarity ${ }^{441}$ 
gives the effect of familiarity on the log odds ratio for detecting a gap. The dB:position and ${ }_{442}$ $d B$ :gap:position parameters correspond to the effect of position within the sequence on false alarm ${ }_{443}$ rate and performance respectively. These are essentially nuisance parameters, but they need to be ${ }_{444}$ included because the intervals are otherwise not independent (the probability of pecking in later ${ }_{445}$ intervals depends on the bird not pecking earlier in the trial). Because the model is nonlinear and ${ }_{446}$ has many interactions, effects and confidence intervals are reported as estimated marginal means, 447 calculated using the emmeans R package (version 1.5.4).

The data for trials with masking noise were analyzed using the same model, but separately. This ${ }^{449}$ was to avoid introducing another interaction into an already complex model.

\section{Extracellular recordings}

Extracellular stimuli The stimuli for the extracellular recordings had the same basic structure, 452 but we allowed the duration of the critical interval to vary and included some additional variants. ${ }^{453}$ Critical intervals were selected to overlap completely with a single syllable in the motif but ${ }_{454}$ were never longer than $100 \mathrm{~ms}$. The design was $2 \times 4$ : two critical intervals per motif, and four 455 variants (Fig. 2B). The variants comprised the continuous stimulus (CS), which was unaltered; the 456 discontinuous stimulus (DS), which replaced the song note within the critical interval with silence; 457 the noise-only stimulus (NS), which was a segment of white noise spanning the critical interval; 458 and the replaced stimulus (RS), which replaced the note within the critical with white noise to 459 produce the illusory perception of the song continuing behind the noise. As with the behavioral 460 stimuli, 2 ms ramps were applied to the edges of the noise and gaps. The noise amplitude was +15461 $\mathrm{dB}$ relative to the motif amplitude and was not varied.

Surgery Birds were anesthetized with isoflurane inhalation (1-3\% in O2) and placed in a stereo- ${ }_{463}$ taxic apparatus (Kopf Instruments). An incision was made in the scalp, and the skin was retracted ${ }_{464}$ from the skull. The recording site was identified using stereotaxic coordinates relative to the Y-sinus. 465 A metal pin was affixed to the skull rostral to the recording site with dental cement, and the skull ${ }_{466}$ over the recording site was shaved down but not completely removed. The bird was allowed to ${ }_{467}$ recover completely for several days prior to recording. 
On the day of recording, the bird was anesthetized with three intramuscular injections of $20 \% \quad 469$ urethane spaced half an hour apart. The bird was placed in a $50 \mathrm{~mL}$ conical tube, and the head 470 pin was attached to a stand in the recording chamber. The thin layer of skull remaining over the ${ }^{471}$ recording site was removed along with the dura, and a well was formed around the recording site 472 and filled with phosphate-buffered saline.

Stimulus presentation Stimuli were presented with the sounddevice python library (version 474 0.3.10) through a Samson Servo 120a amplifier to a Behringer Monitor Speaker 1C. The RMS 475 amplitude of the unmodified motifs was $70 \mathrm{~dB}$ SPL. Stimuli were presented in a pseudorandom 476 order to minimize stimulus adaptation, with $1 \mathrm{~s}$ between each song. Each stimulus was presented 477 10 times.

Data acquisition Neural recordings were made using a NeuroNexus 32-channel probe in a 479 four-shank, linear configuration (A4x8-5mm-100-400-177-A32) connected to an Intan RHD2132 480 Amplifier Board. Data were collected by the Open Ephys Acquisition Board and sent to a computer ${ }_{481}$ running Open Ephys GUI software (version 0.4.6).

The recording electrode was coated with DiI (Invitrogen). The electrode was inserted at a dorso- ${ }^{483}$ rostral to ventro-caudal angle that allowed for recording of all auditory forebrain regions with a ${ }_{484}$ single penetration (Fig. 2A). The probe was lowered into the brain until the local field potentials ${ }_{485}$ across channels and shanks showed coordinated responses to birdsong, and the probe was allowed 486 to rest in place for half an hour to ensure a stable recording. Recordings of responses were made ${ }^{487}$ across all 32 channels. After the recording, the probe was moved to successively deeper regions of ${ }_{488}$ the auditory pathway and additional recordings were made.

Histology After recording, birds were administered a lethal intramuscular injection of Eutha- 490 sol and perfused transcardially with a $10 \mathrm{U} / \mathrm{mL}$ solution of sodium heparin in PBS (in mM: 10491 Na2HPO4, $154 \mathrm{NaCl}, \mathrm{pH}$ 7.4) followed by 4\% formaldehyde (in PBS). Brains were immediately ${ }_{492}$ removed from the skull, postfixed overnight in $4 \%$ formaldehyde at $4{ }^{\circ} \mathrm{C}$, cryoprotected in $30 \% \quad 493$ sucrose (in 100 mM Na2HPO4, pH 7.4), blocked saggitally into hemispheres or on a modified ${ }_{494}$ 
coronal plane (Chen and Meliza, 2018), embedded in OCT, and stored at $-80{ }^{\circ} \mathrm{C} .60 \mu \mathrm{m}$ sections ${ }^{495}$ were cut on a cryostat and mounted on slides. After drying overnight, the sections were rehy- 496 drated in PBS and coverslipped with Prolong Gold with DAPI (ThermoFisher, catalog P36934; 497 RRID:SCR_015961). Sections were imaged using epifluorescence with DAPI and Texas Red filter 498 cubes to located DiI-labeled penetrations. Images of the electrode tracks were used to identify the ${ }_{499}$ locations of recorded units (Fig. 2A).

Spike sorting Spikes were sorted offline using MountainSort 4. Single units were further curated 501 by visual inspection for spheroid PCA cluster shape, very low refractory period violations in the 502 autocorrelogram, and stability of the unit throughout the recording. These high-quality single units 503 were included in the dataset if they showed a clear, phase-locked auditory response to at least one 504 stimulus.

\section{Stimulus reconstruction}

A linear stimulus decoding model was used to predict stimulus spectrograms from recorded neural 507 responses (Mesgarani et al., 2009; Crosse et al., 2016; Leonard et al., 2016). The model is similar to 508 the spectrotemporal receptive field (STRF), in which the expected firing rate of a single neuron at a 509 given time point $t$ is modeled as a linear function of the stimulus spectrogram immediately prior to 510 $t$. In the linear decoding model, the relationship is reversed, and the expected stimulus at time $t$ is 511 modeled as a linear function of the response that follows. Using a discrete time notation where $s_{t}$ is 512 the stimulus in the time bin around $t$, and $r_{t}$ is the response of a single neuron in the same time bin, 513 then the expected value of the stimulus is given by

$$
\mathrm{E}\left(s_{t}\right)=r_{t} g_{0}+r_{t+1} g_{1}+\cdots+r_{t+k} g_{k}
$$

where $k$ is the number of time bins one looks into the future, and $\mathbf{g}=\left(g_{0}, g_{1}, \ldots, g_{k}\right)$ are the 515 linear coefficients of the model. If the errors are independent and normally distributed around the 516 expectation with constant variance $\sigma^{2}$, then this is a ordinary linear model. If there are $n$ time bins 517 
in the stimulus, then the stimulus is a vector $\mathbf{s}=\left(s_{0}, \ldots, s_{n}\right)$ drawn from a multivariate normal 518 distribution. In vector notation,

$$
\mathbf{s} \mid \mathbf{g}, \sigma^{2}, \mathbf{R} \sim N\left(\mathbf{R} \mathbf{g}, I \sigma^{2}\right)
$$

where $\mathbf{R}$ is the $n \times k$ Hankel matrix of the response. Without any loss of generality, the model can 520 be expanded to include the responses of multiple neurons. If there are $p$ neurons, then $r_{t}$ becomes a ${ }_{521}$ $p$-element vector $\left(r_{1, t}, \ldots, r_{p, t}\right), \mathbf{R}$ becomes a $n \times p k$ matrix formed by concatenating the Hankel 522 matrices for each of the neurons, and $\mathbf{g}$ becomes a $p k$-element vector.

Because the model is simply linear regression, standard tools can be used to estimate the parameters 524 $\mathbf{g}$ and $\sigma^{2}$. Using a ridge penalty for regularization, the maximum likelihood estimate of $\mathbf{g}$ is ${ }_{525}$

$$
\hat{\mathbf{g}}=\left(\mathbf{R}^{\top} \mathbf{R}+\lambda \mathbf{I}\right)^{-1} \mathbf{R}^{\top} \mathbf{s}
$$

where $\lambda \mathbf{I}$ is the identity matrix multiplied by the shrinkage penalty for the ridge regression.

Additional regularization can be achieved by projecting the response matrix $\mathbf{R}$ into an alternative ${ }_{527}$ basis set, such as a non-linearly spaced series of raised cosines (Pillow et al., 2005). The width of 528 each basis function increases with lag, which gives the model high temporal resolution at short ${ }_{529}$ lags and lower resolution at longer lags. This allows the inclusion of longer lags without exploding 530 the number of parameters.

In this study, the response matrix was constructed from the peristimulus time histograms (PSTHs) 532 averaged over 10 trials for each of the 407 auditory single units from all 14 birds, using a bin size of 533 $1 \mathrm{~ms}$. Stimuli were converted to time-frequency representations using a gammatone filter bank, 534 implemented in the Python package gammatone (version 1.0) with 50 log-spaced frequency bands 535 from 1-8 kHz, a window size of $2.5 \mathrm{~ms}$, and a step size of $1 \mathrm{~ms}$. Power was log-transformed with a ${ }_{536}$ constant offset of 1 , giving the transformed signal a lower bound of $0 \mathrm{~dB}$. 
The parameters were estimated with data from the Continuous (CS), Discontinuous (DS), and 538 Noise-only (NS) stimuli, leaving out the Replaced (RS) responses for prediction, using the Python 539 machine-learning library scikit-learn (version 0.23.0). We combined all 50 spectral bands into a 540 single multivariate multiple regression, and then used 4-fold cross-validation to determine the ${ }_{541}$ best values for the ridge penalty $\lambda$, the number of time lags $k$, the number of raised-cosine basis 542 functions, and a linearity parameter that controls the spacing of the basis functions. Basis functions 543 were defined as in Pillow et al. (2005). Because the models differed in the number of parameters, 544 Aikake information criteron (AIC) was used for scoring. For the reconstructions presented here, 545 the optimal value for $\lambda$ was $8.59, k$ was 300 , the number of basis functions was 30 , and the linearity 546 factor was 30 .

After fitting the model, we used the parameter estimates to decode the stimulus from the responses 548 to the RS stimuli. Using $\tilde{\mathbf{R}}$ to denote these responses, the predicted stimulus is calculated as

$$
\mathrm{E}(\tilde{\mathbf{S}})=\tilde{\mathbf{R}} \hat{\mathbf{g}}
$$

To quantify how similar the decoded stimulus was to the actual stimulus and the other variants, 550 we calculated the correlation coefficient between $\tilde{\mathbf{S}}$ and $\mathbf{S}_{C S}, \mathbf{S}_{R S}, \mathbf{S}_{D S}$, and $\mathbf{S}_{C+N}$ within the critical 551 interval for each of the motifs. $\mathbf{S}_{C+N}$ comprised a 1:1 mixture of $\mathbf{S}_{C S}$ and $\mathbf{S}_{R S}$, representing the ${ }_{552}$ expected illusory percept. As a baseline for how good the reconstruction could be, we calculated 553 the correlation between $\tilde{\mathbf{S}}$ and $\mathbf{S}_{R S}$ in an interval outside the critical interval (which was the same ${ }_{554}$ for all variants).

Unless otherwise noted, all comparisons were fit with a mixed-effects linear model using lme4 556 (version 1.1-23) in R and emmeans (version 1.5.2-1) was used to calculate the effects, confidence 557 intervals, and significance. 
Spectrogram correlations are a simple and easily interpretable way to quantify the similarity of 560 the decoded stimulus to the physical stimulus and the illusion, but what we really want to know 561 is, given the response to RS, how likely is it that the stimulus included the missing syllable? 562 Equivalently, how much evidence is there in the neural response that the missing syllable was 563 present? To answer this question, we used the decoding model to compute the posterior probability 564 that the predicted stimulus $\mathrm{E}(\tilde{\mathbf{S}})$ was the expected illusory percept $\mathbf{S}_{C+N}$, relative to the probability 565 that it was the actual stimulus physically presented to the animal $\mathbf{S}_{R S}$.

For a linear regression model, the posterior predictive distribution conditional on the observations 567 used to fit the model (Gelman et al., 2020) is a multivariate $t$ distribution with a mean given by 568 Equation (1), $n-k$ degrees of freedom, and scale matrix $s^{2}\left(\mathbf{I}+\tilde{\mathbf{R}} \mathbf{V}_{g} \tilde{\mathbf{R}}^{\top}\right)$, where $s^{2}$ is the sample ${ }_{569}$ variance of the residuals,

$$
s^{2}=\frac{1}{n-k}(\mathbf{S}-\mathbf{R} \hat{\mathbf{g}})^{\top}(\mathbf{S}-\mathbf{R} \hat{\mathbf{g}}),
$$

and $\mathbf{V}_{\mathbf{g}}$ is the posterior variance of the parameter estimates,

$$
\mathbf{V}_{\mathbf{g}}=\left(\mathbf{R}^{\top} \mathbf{R}\right)^{-1}
$$

Thus, the posterior predictive uncertainty reflects both the unexplained variance in the model 572 $\left(s^{2}\right)$ and the posterior uncertainty in the parameter estimates $\left(\mathbf{V}_{\mathbf{g}}\right)$. Because $n-k$ was very large ${ }_{573}$ $(27,725)$, we approximated this distribution with a multivariate normal with mean $\mathbf{R} \hat{\mathbf{g}}$ and variance 574 $s^{2}$ and calculated $\operatorname{Pr}\left(\tilde{\mathbf{S}}=\mathbf{S}_{C+N} \mid \mathbf{S}\right)$ and $\operatorname{Pr}\left(\tilde{\mathbf{S}}=\mathbf{S}_{x} \mid \mathbf{S}\right)$. These probabilities (or likelihoods) are not 575 meaningful on their own, but the $\log$ of their ratio $(\ell)$ quantifies how much more likely it was (given 576 the response) that the stimulus was the combined continuous and noise stimulus $(\mathrm{C}+\mathrm{N})$ or just the 577 noise (RS). By analogy to Bayes Factors, this ratio can also be thought of as the amount of evidence 578 
in the neural response for the presence of the missing syllable. Because $p(\tilde{\mathbf{S}} \mid \mathbf{S})$ is multivariate ${ }_{579}$ normal, with each dimension corresponding to each time-frequency point in the stimulus, we can 580 either evaluate the marginal likelihood for each point separately to produce a spectrotemporal 581 evidence plot (Fig. 3A) or use the joint distribution to obtain a single value of $\ell$ for each critical 582 interval (Fig. 3B). Note that outside the critical interval, $\ell$ is always zero because $\mathbf{S}_{R S}$ and $\mathbf{S}_{C+N}$ are ${ }_{583}$ identical.

\section{Area-level analysis}

To understand how different auditory areas in the zebra finch auditory cortex influence the overall 586 reconstruction, we performed a leave-one-area-out analysis. Using the same cross-validated ${ }_{587}$ parameters as the full reconstruction, we fit the model to the entire dataset minus the units from one 588 area. This technique allowed us to identify unique contributions made by each of the auditory area 589 because if the responses of one area were redundant with those of another, the model would shift its 590 weights but the estimated reconstruction would remain unchanged. Changes in the reconstruction 591 seen with this method mean that the model lost irreplaceable evidence when the units from one 592 area were omitted.

To quantify the changes in reconstructions, we calculated the log likelihood ratio between $\operatorname{Pr}(\tilde{\mathbf{S}}=594$ $\left.\mathbf{S}_{C+N} \mid \mathbf{S}\right)$ and $\operatorname{Pr}\left(\tilde{\mathbf{S}}=\mathbf{S}_{R S} \mid \mathbf{S}\right)$, using the area-specific reconstructions. We used the joint distribution 595 and compared this value $\ell^{(i)}$ to the $\log$ likelihood ratio $\ell$ for the full model. A decrease in $\ell$ relative to 596 the full model therefore implied that the removed area caused $\operatorname{Pr}\left(\tilde{\mathbf{S}}=\mathbf{S}_{C+N} \mid \mathbf{S}\right)$ to increase relative ${ }_{597}$ to $\operatorname{Pr}\left(\tilde{\mathbf{S}}=\mathbf{S}_{R S} \mid \mathbf{S}\right)$, and that neurons in that area were non-redundantly contributing evidence for 598 the missing syllable.

\section{Acknowledgments}

We would like to thank Samantha Moseley for assistance with the behavioral experiment, Leah ${ }_{601}$ Kiely for assistance with histology, and Ayush Sagar and Crystal Gong for their work developing 602 and testing the operant apparatus used in this study. This work was supported by NSF IOS-1942480 603 and NIH R01DC018621 


\section{References}

Bashford JA, Warren RM. Multiple phonemic restorations follow the rules for auditory induction. 606 Percept Psychophys 1987 Aug;42(2):114-121. doi: 10.3758/bf03210499.

Braaten RF, Leary JC. Temporal Induction of Missing Birdsong Segments in European Starlings. 608 Psychological Science 1999 May;10(2):162-166. doi: 10.1111/1467-9280.00125.

Bradlow AR, Kraus N, Hayes E. Speaking clearly for children with learning disabilities: sen- 610 tence perception in noise. J Speech Lang Hear Res 2003 Feb;46(1):80-97. doi: 10.1044/1092- 611 4388(2003/007).

Braida LD, Lim JS, Berliner JE, Durlach NI, Rabinowitz WM, Purks SR. Intensity perception. XIII. ${ }_{613}$ Perceptual anchor model of context-coding. The Journal of the Acoustical Society of America 614 1998 Jun;76(3):722. doi: 10.1121/1.391258.

Bregman AS. Auditory scene analysis. 2nd edition ed. The perceptual organization of sound, MIT ${ }_{616}$ Press; 1999.

Calabrese A, Woolley SMN. Coding principles of the canonical cortical microcircuit in the avian ${ }_{618}$ brain. PNAS 2015 Mar;112(11):3517-3522. doi: 10.1073/pnas.1408545112.

Chen AN, Meliza D. Phasic and Tonic Cell Types in the Zebra Finch Auditory Caudal Mesopallium. 620 J Neurophys 2018 Mar;119(3):1127-1139. doi: 10.1152/jn.00694.2017.

Crosse MJ, Di Liberto GM, Bednar A, Lalor EC. The Multivariate Temporal Response Function 622 (mTRF) Toolbox: A MATLAB Toolbox for Relating Neural Signals to Continuous Stimuli. Front ${ }_{623}$ Hum Neurosci 2016;10:604. doi: 10.3389/fnhum.2016.00604.

Elie JE, Theunissen FE. The vocal repertoire of the domesticated zebra finch: a data-driven approach ${ }_{625}$ to decipher the information-bearing acoustic features of communication signals. Anim Cogn ${ }_{626}$ 2016 Mar;19(2):285-315. doi: 10.1007/s10071-015-0933-6.

Gelman A, Carlin JB, Stern HS, Dunson DB, Vehtari A, Rubin DB. Introduction to regression models. ${ }_{628}$ In: Bayesian Data Analysis Chapman \& Hall; 2020.p. 353-378. 
Gilbert CD, Sigman M. Brain states: top-down influences in sensory processing. Neuron 2007630 Jun;54(5):677-696. doi: 10.1016/j.neuron.2007.05.019.

Glaser JI, Benjamin AS, Chowdhury RH, Perich MG, Miller LE, Kording KP. Machine Learning for 632 Neural Decoding. eneuro 2020 Jul;7(4). doi: 10.1523/ENEURO.0506-19.2020.

Heald SLM, Nusbaum HC. Speech perception as an active cognitive process. Front Syst Neurosci ${ }_{634}$ 2014;8:35. doi: 10.3389/fnsys.2014.00035.

Ishida M, Arai T. Missing phonemes are perceptually restored but differently by native and ${ }_{636}$ non-native listeners. Springerplus 2016;5(1):713-10. doi: 10.1186/s40064-016-2479-8.

Komatsu H. The neural mechanisms of perceptual filling-in. Nat Rev Neurosci 2006 Mar;7(3):220- ${ }^{638}$ 231. doi: $10.1038 / \operatorname{nrn} 1869$.

Leonard MK, Baud MO, Sjerps MJ, Chang EF. Perceptual restoration of masked speech in human 640 cortex. Nat Commun 2016 Dec;7(1):13619-9. doi: 10.1038/ncomms13619.

Liberman AM, Mattingly IG. The motor theory of speech perception revised. Cognition 1985642 Oct;21(1):1-36.

McClelland JL, Elman JL. The TRACE model of speech perception. Cogn Psychol 1986;18(1):1-86. ${ }^{644}$

Meliza CD, Margoliash D. Emergence of selectivity and tolerance in the avian auditory cortex. J ${ }_{645}$ Neurosci 2012 Oct;32(43):15158-15168. doi: 10.1523/JNEUROSCI.0845-12.2012.

Mesgarani N, David SV, Fritz JB, Shamma SA. Influence of context and behavior on stimulus ${ }_{647}$ reconstruction from neural activity in primary auditory cortex. J Neurophys 2009 Dec;102(6):3329- 648 3339. doi: 10.1152/jn.91128.2008.

Miller CT, Dibble E, Hauser MD. Amodal completion of acoustic signals by a nonhuman primate. 650 Nat Neurosci 2001 Aug;4(8):783-784. doi: 10.1038/90481.

Miller GA, Licklider JCR. The Intelligibility of Interrupted Speech. J Acoust Soc Am 1950652 Jun;22(2):167-173. doi: 10.1121/1.1906584.

Narayan R, Best V, Ozmeral E, McClaine E, Dent ML, Shinn-Cunningham B, et al. Cortical ${ }_{654}$ interference effects in the cocktail party problem. Nat Neurosci 2007;10(12):1601-1607. 
Petkov CI, O'Connor KN, Sutter ML. Illusory sound perception in macaque monkeys. J Neurosci 656 2003 Oct;23(27):9155-9161. doi: 10.1523/JNEUROSCI.23-27-09155.2003.

Petkov CI, O'Connor KN, Sutter ML. Encoding of illusory continuity in primary auditory cortex. 658 Neuron 2007 Apr;54(1):153-165. doi: 10.1016/j.neuron.2007.02.031.

Petkov CI, Sutter ML. Evolutionary conservation and neuronal mechanisms of auditory perceptual 660 restoration. Hear Res 2011 Jan;271(1-2):54-65. doi: 10.1016/j.heares.2010.05.011.

Pillow JW, Paninski L, Uzzell VJ, Simoncelli EP, Chichilnisky EJ. Prediction and Decoding of Retinal ${ }_{662}$ Ganglion Cell Responses with a Probabilistic Spiking Model. J Neurosci 2005 Nov;25(47):11003- ${ }_{663}$ 11013. doi: 10.1523/JNEUROSCI.3305-05.2005.

Rao RP, Ballard DH. Predictive coding in the visual cortex: a functional interpretation of some ${ }_{665}$ extra-classical receptive-field effects. Nat Neurosci 1999 Jan;2(1):79-87. doi: 10.1038/4580. ${ }_{666}$

Samuel AG. Does lexical information influence the perceptual restoration of phonemes? J Exp ${ }_{667}$ Psychol Gen 1996;125(1):28-51. doi: 10.1037/0096-3445.125.1.28. ${ }_{668}$

Schneider DM, Woolley SMN. Sparse and background-invariant coding of vocalizations in auditory ${ }_{669}$ scenes. Neuron 2013 Jul;79(1):141-152. doi: 10.1016/j.neuron.2013.04.038. 670

Seeba F, Klump GM. Stimulus familiarity affects perceptual restoration in the European starling ${ }^{671}$ (Sturnus vulgaris). PLoS ONE 2009 Jun;4(6):e5974. doi: 10.1371/journal.pone.0005974. 672

Seeba F, Schwartz JJ, Bee MA. Testing an auditory illusion in frogs: Perceptual restoration or 673 sensory bias? Anim Behav 2010 Jun;79(6):1317-1328. doi: 10.1016/j.anbehav.2010.03.004. 674

Sen K, Theunissen FE, Doupe AJ. Feature analysis of natural sounds in the songbird auditory 675 forebrain. J Neurophysiol 2001 Sep;86(3):1445-1458. 676

Singh NC, Theunissen FE. Modulation spectra of natural sounds and ethological theories of 677 auditory processing. J Acoust Soc Am 2003 Dec;114(6):3394-3411. 678

Sugita Y. Neuronal correlates of auditory induction in the cat cortex. Neuroreport 1997679 Mar;8(5):1155-1159. doi: 10.1097/00001756-199703240-00019. 
Wang Y, Brzozowska-Prechtl A, Karten HJ. Laminar and columnar auditory cortex in avian brain. ${ }_{681}$ PNAS 2010 Jul;107(28):12676-12681. doi: 10.1073/pnas.1006645107.

Warren RM. Perceptual restoration of missing speech sounds. Science 1970 Jan;167(3917):392-393. 683 doi: 10.1126 /science.167.3917.392.

Warren RM, Sherman GL. Phonemic restorations based on subsequent context. Percept Psychophys 685 1974 Jan;16(1):150-156. doi: 10.3758/BF03203268.

Zokoll MA, Klump GM, Langemann U. Auditory short-term memory persistence for tonal signals ${ }_{687}$ in a songbird. J Acoust Soc Am 2007 May;121(5):2842. doi: 10.1121/1.2713721. 
bioRxiv preprint doi: https://doi.org/10.1101/2021.07.19.452925; this version posted July 19, 2021. The copyright holder for this preprint (which

was not certified by peer review) is the author/funder, who has granted bioRxiv a license to display the preprint in perpetuity. It is made available under aCC-BY-NC-ND 4.0 International license.

Bjoring and Meliza - Neural reconstruction of occluded song (preprint)

p. 31

\section{Supplementary Materials}

Supplementary Figures 1-5

Supplementary Table 1

Supplementary Movie 1 
bioRxiv preprint doi: https://doi.org/10.1101/2021.07.19.452925; this version posted July 19, 2021. The copyright holder for this preprint (which was not certified by peer review) is the author/funder, who has granted bioRxiv a license to display the preprint in perpetuity. It is made available under aCC-BY-NC-ND 4.0 International license.
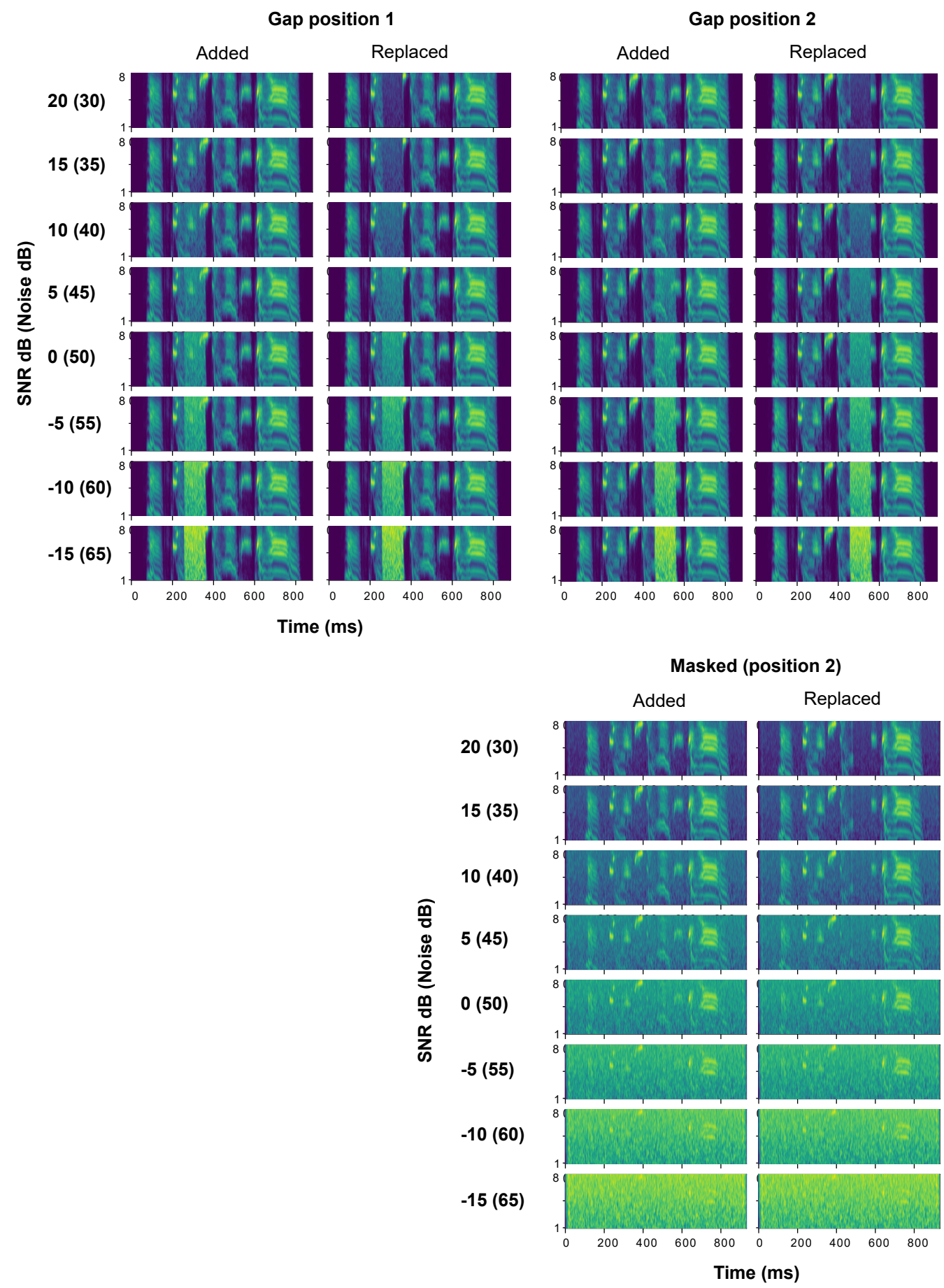

Supplementary Fig. 1. All the stimulus variants for a single motif. 
bioRxiv preprint doi: https://doi.org/10.1101/2021.07.19.452925; this version posted July 19, 2021. The copyright holder for this preprint (which was not certified by peer review) is the author/funder, who has granted bioRxiv a license to display the preprint in perpetuity. It is made available under aCC-BY-NC-ND 4.0 International license.

Bjoring and Meliza - Neural reconstruction of occluded song (preprint)

p. 33

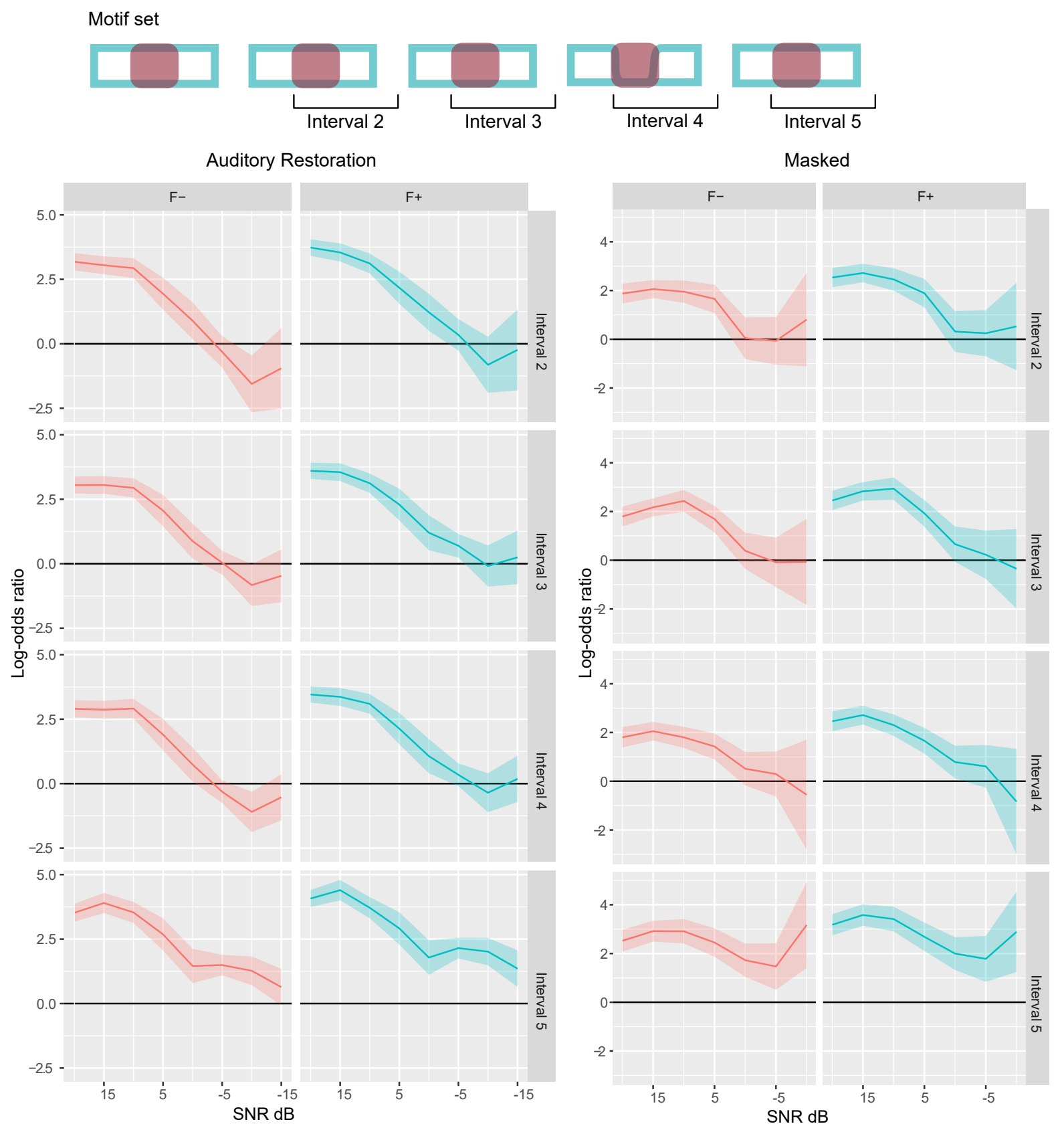

Supplementary Fig. 2. GLMM estimates of performance for all intervals. Top: An example motif sequence illustrating different response intervals. In this example, a peck during interval 4 would be a correct detection, and a false alarm in any other interval. Left: GLMM estimates and 90\% confidence intervals for the auditory restoration task faceted by interval and familiarity (F-: unfamiliar; F+: familiar). The increase in performance in interval 5 likely indicates both a perceptual anchoring effect and a learned guessing strategy for the last motif in the sequence. Right: GLMM estimates and $90 \%$ confidence intervals for the masked task. 
bioRxiv preprint doi: https://doi.org/10.1101/2021.07.19.452925; this version posted July 19,2021. The copyright holder for this preprint (which was not certified by peer review) is the author/funder, who has granted bioRxiv a license to display the preprint in perpetuity. It is made available under aCC-BY-NC-ND 4.0 International license.
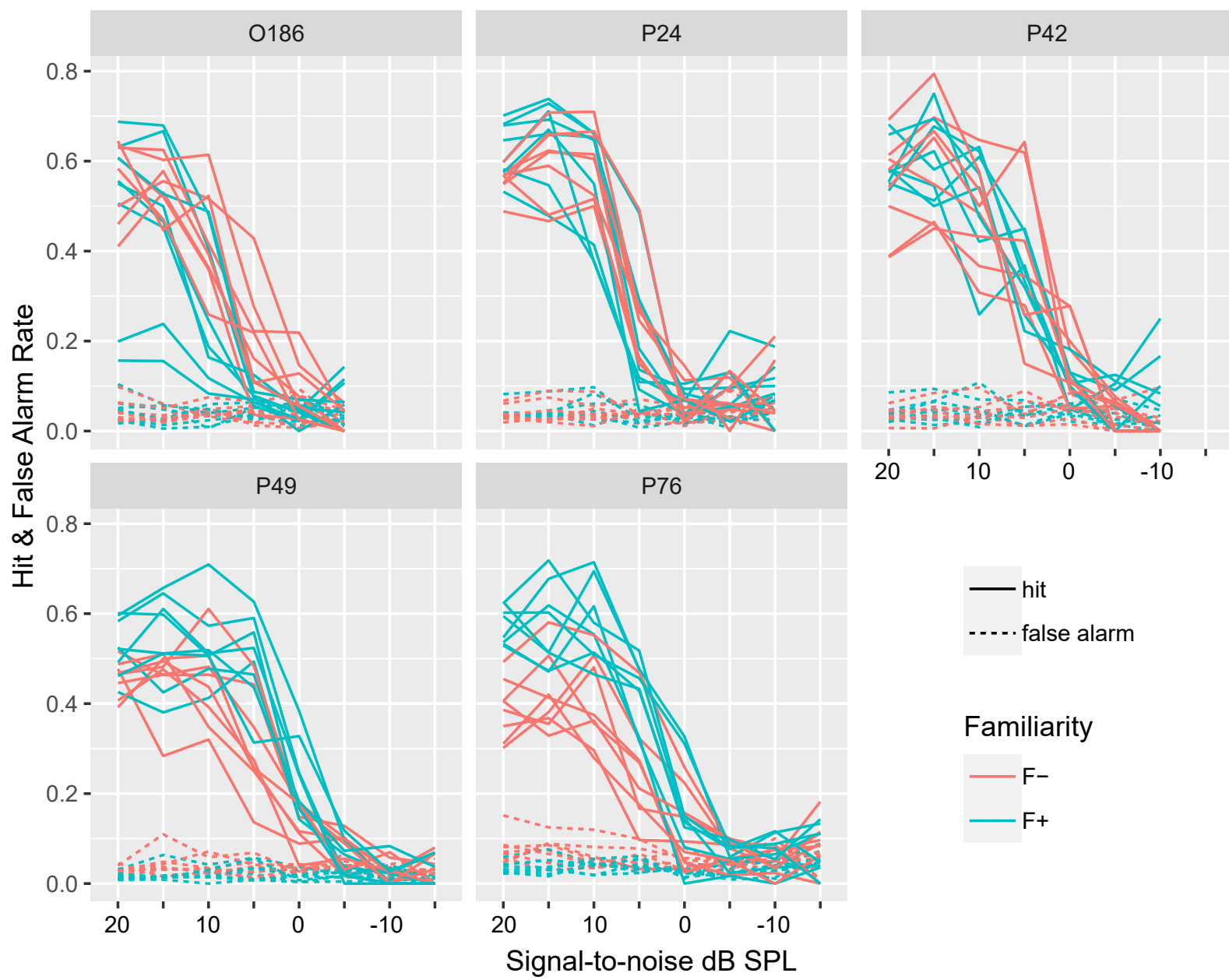

\section{Familiarity}

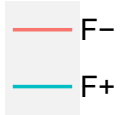

Supplementary Fig. 3. Within-subject variability. Hit and false alarm rates for each subject, with each trace corresponding to one motif variant and color indicating familiarity. 
bioRxiv preprint doi: https://doi.org/10.1101/2021.07.19.452925; this version posted July 19, 2021. The copyright holder for this preprint (which was not certified by peer review) is the author/funder, who has granted bioRxiv a license to display the preprint in perpetuity. It is made available under aCC-BY-NC-ND 4.0 International license.

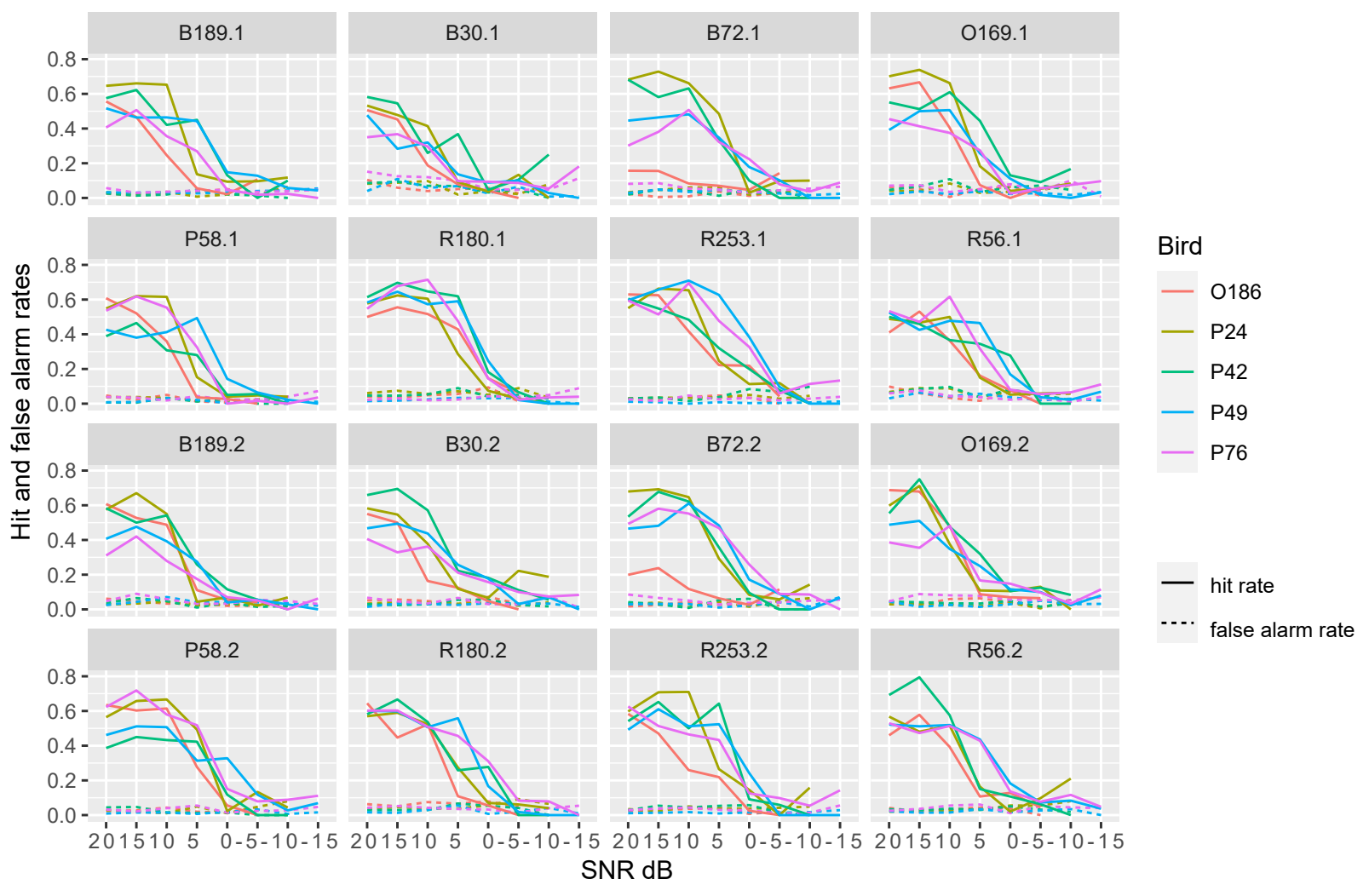

Supplementary Fig. 4. Between-subject variability. Hit and false alarm rates for each motif variant, with each trace corresponding to one subject. 
bioRxiv preprint doi: https://doi.org/10.1101/2021.07.19.452925; this version posted July 19, 2021. The copyright holder for this preprint (which was not certified by peer review) is the author/funder, who has granted bioRxiv a license to display the preprint in perpetuity. It is made available under aCC-BY-NC-ND 4.0 International license.

Bjoring and Meliza - Neural reconstruction of occluded song (preprint)

p. 36

a

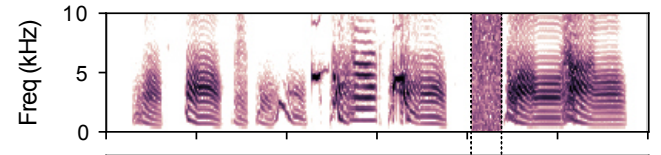

CS

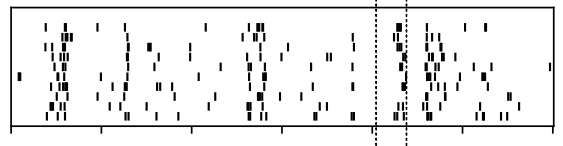

DS

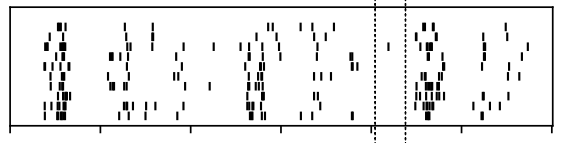

NS

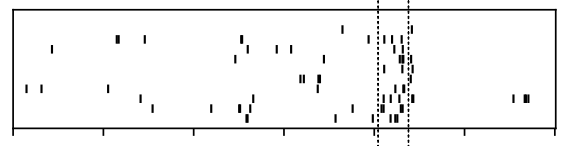

RS

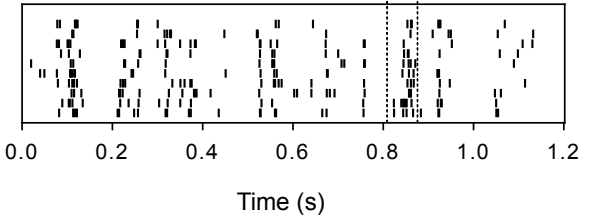

b
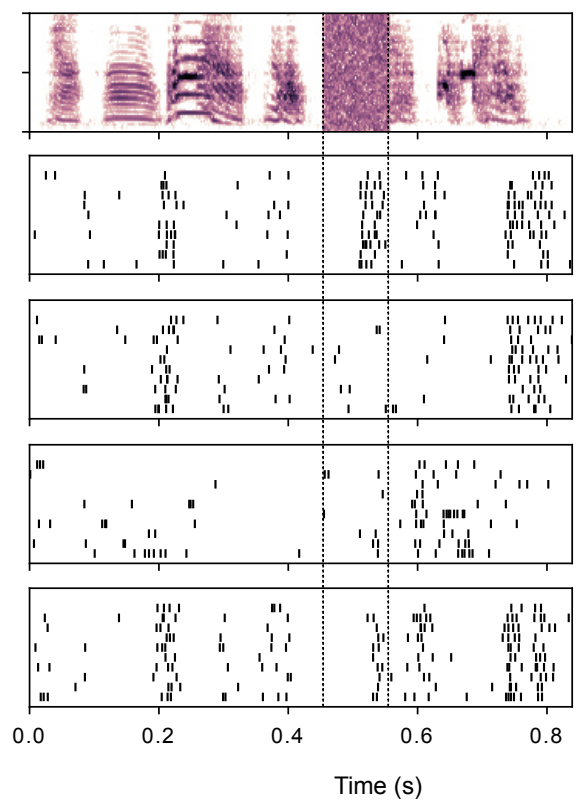

C
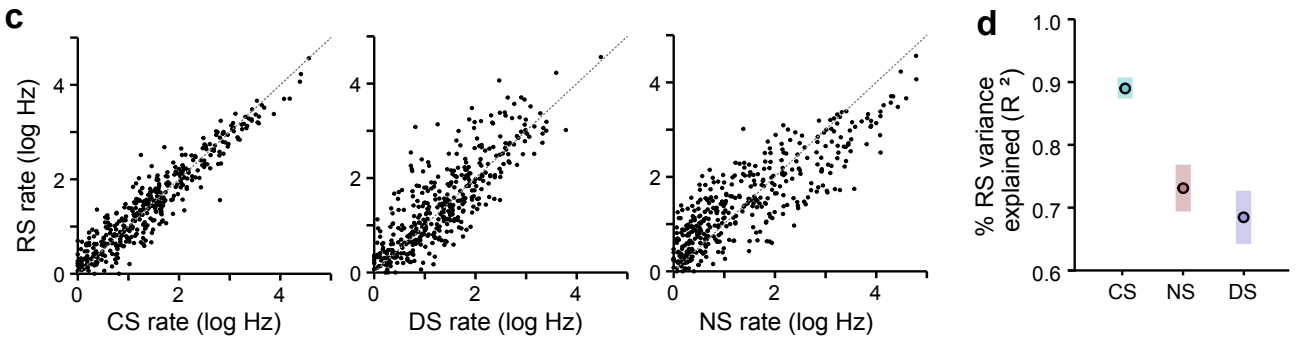

Supplementary Fig. 5. Single-unit responses to occluded motifs. (a) Raster plot of an example single unit responding to the continuous (CS), discontinous (DS), replaced (RS) and noise (NS) variants of a motif. Top spectrogram shows the RS variant. Dotted lines indicate the critical interval. Note that the response to RS during the critical interval is more similar to CS than to DS or NS. (b) Raster plot of another example single unit, same format as a. (c) Average firing rates (log scale) during the critical interval of RS for all units compared to response rates to CS, DS, and NS during the same interval. Each point corresponds to an individual neuron $(n=407)$, and the dotted line indicates equality. CS response rates are highly predictive of RS response rates, while the rates for DS and NS show more scatter. (d) $R^{2}$ values (black dots) and 90\% confidence intervals (colored bars) for linear regressions of RS firing rates against CS, NS, and DS rates. 
bioRxiv preprint doi: https://doi.org/10.1101/2021.07.19.452925; this version posted July 19, 2021. The copyright holder for this preprint (which was not certified by peer review) is the author/funder, who has granted bioRxiv a license to display the preprint in perpetuity. It is made available under aCC-BY-NC-ND 4.0 International license.

Bjoring and Meliza - Neural reconstruction of occluded song (preprint)

p. 37

Supplementary Table 1. Trial numbers per block during task-specific training.

\begin{tabular}{lllllll}
\hline Subject & 3 Motifs & 4 Motifs & 5 Motifs & Ending LOR & Sex & Group \\
\hline O186† & 3268 & 3044 & 839 & $1.15 \pm 0.19$ & M & B \\
P17 & 2703 & 8193 & 12340 & $1.24 \pm 0.19$ & F & B \\
P24 & 2832 & 10195 & 13344 & $1.02 \pm 0.26$ & M & B \\
P29 & 2807 & 13371 & $12588^{*}$ & & M & A \\
P30 & 1360 & $6104^{*}$ & & & M & A \\
P35 & 2155 & 7671 & $3923^{*}$ & & M & B \\
P42 & 1876 & 6772 & 10203 & $1.20 \pm 0.22$ & F & B \\
P49† & 10375 & 2237 & 9327 & $1.20 \pm 0.17$ & F & A \\
P52† & 191 & $687 \ddagger$ & & & F & A \\
P76† & 3731 & 2063 & 1729 & $1.06 \pm 0.19$ & M & A \\
P8† & 4980 & $3920^{*}$ & & & F & A \\
\hline
\end{tabular}

*bird was excluded during this stage

tbird was previously trained on a similar same-different task

fbird was excluded for low trial initiation

Movie 1. Well-trained zebra finch running trials to detect motifs with gaps in a sequence of 694 otherwise identical motifs. 